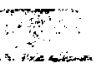

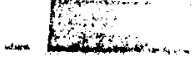

LBL --25618

DE91 004139

\title{
Coupled transport processes in semipermeable media.
} Part I: Theoretical basis

\author{
C. L. Carnahan and J. S. Jacobsen
}

Earth Sciences Division, Lawrence Berkeley Laboratory

University of California, Berkeley, California

April 1990

This work was supported by the Director, Office of Energy Research, Office of Basic Energy Sciences, Engineering and Geosciences Division, of the U. S. Department of Energy under Contract No. DE-AC0376SF00098. 


\section{DISCLAIMER}

This report was prepared as an account of work sponsored by an agency of the United States Government. Neither the United States Government nor any agency Thereof, nor any of their employees, makes any warranty, express or implied, or assumes any legal liability or responsibility for the accuracy, completeness, or usefulness of any information, apparatus, product, or process disclosed, or represents that its use would not infringe privately owned rights. Reference herein to any specific commercial product, process, or service by trade name, trademark, manufacturer, or otherwise does not necessarily constitute or imply its endorsement, recommendation, or favoring by the United States Government or any agency thereof. The views and opinions of authors expressed herein do not necessarily state or reflect those of the United States Government or any agency thereof. 


\section{DISCLAIMER}

Portions of this document may be illegible in electronic image products. Images are produced from the best available original document. 



\section{TABLE OF CONTENTS}

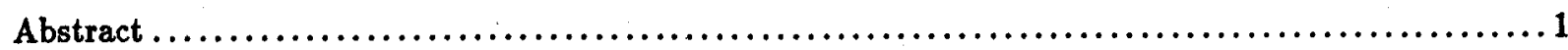

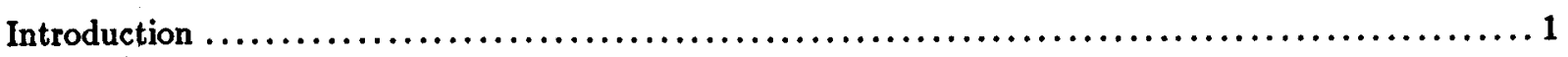

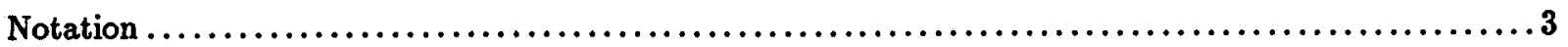

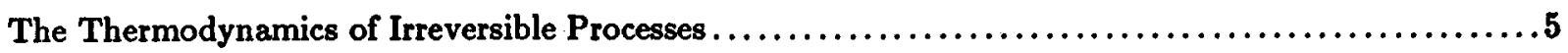

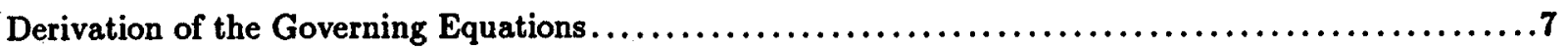

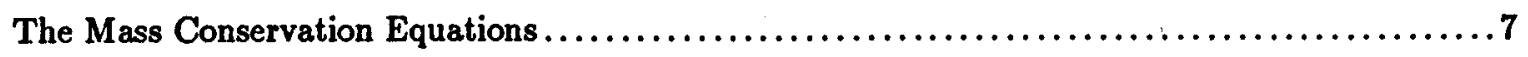

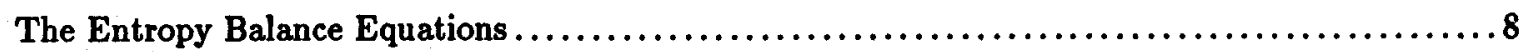

Balance Equations for Intensive State Variables................................

Introduction of the Gibbs Equation and Other Conservation Equations $\ldots \ldots \ldots \ldots \ldots \ldots \ldots \ldots \ldots$

Introduction of the Volume Flux and Diffusional Solute Fluxes.......................11

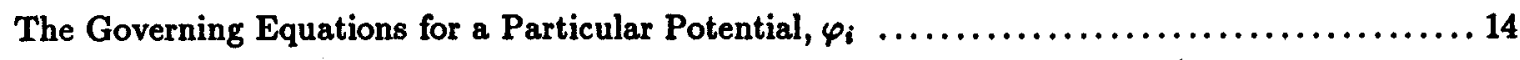

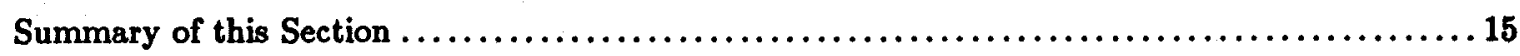

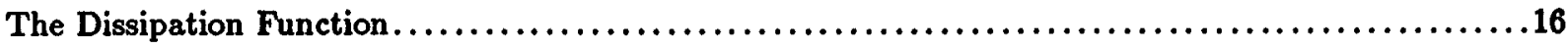

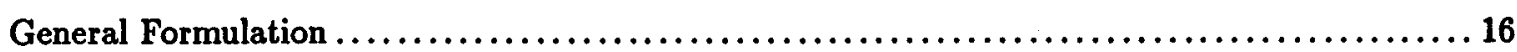

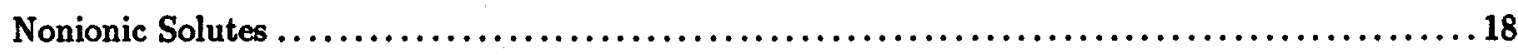

Ionic Solutes with Electroneutrality and Zero Ionic Current $\ldots \ldots \ldots \ldots \ldots \ldots \ldots \ldots \ldots \ldots$

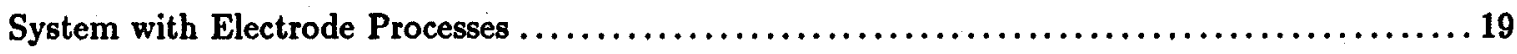

The Chemical Affinities .................................................... 19

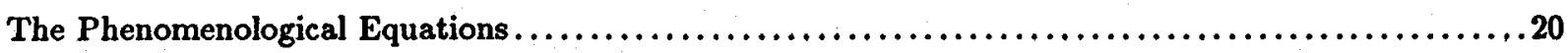

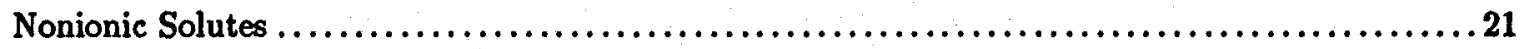

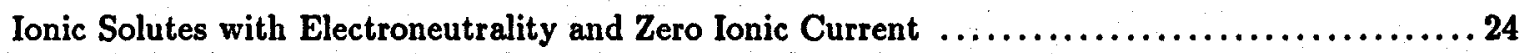

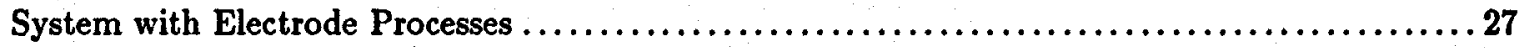

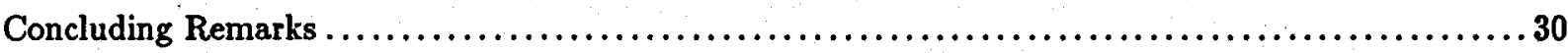

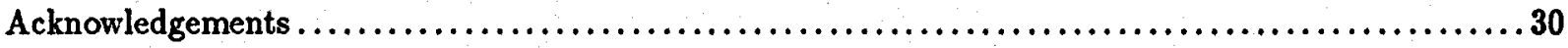

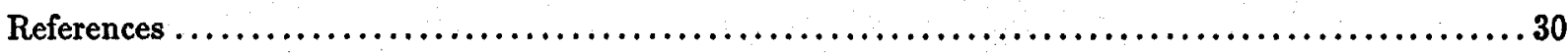

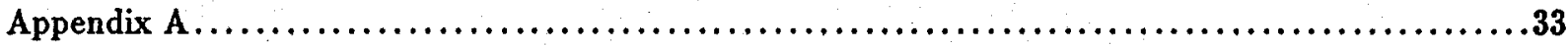

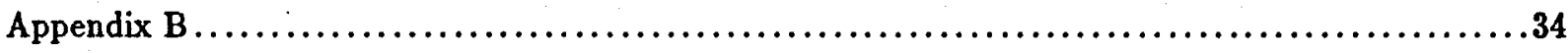

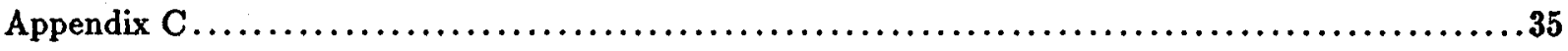




\begin{abstract}
The thermodynamics of irreversible processes (TTIP) is used to derive governing equations and phenomenological equations for transport processes and chemical reactions in water-saturated semipermeable media. TTIP is based on three fundamental postulates. The first postulate, the assumption of local equilibrium, allows the formulation of balance equations for entropy. These equations are the bases for the derivation of governing equations for the thermodynamic variables, temperature, pressure, and composition. The governing equations involve vector fluxes of heat and mass and scalar rates of chemical reactions; in accordance with the second postulate of TTIP, these fluxes and rates are related, respectively, to all vector and all scalar driving forces (gradients of thermodynamic variables) acting within the system. The third postulate of TTIP states equality (the Onsager reciprocal relations) between certain of the phenomenological coefficients relating forces and fluxes. The description by TTIP of a system undergoing irreversible processes allows consideration of coupled transport processes such as thermal osmosis, chemical osmosis, and ultrafiltration. The coupled processes can make significant contributions to flows of mass and energy in slightly permeable, permselective geological materials such as clays and shales.
\end{abstract}

\title{
INTRODUCTION
}

Coupled processes can be classified generally in two categories. In one category, spatially and temporally varying fields of thermodynamic potentials can interact with physical, chemical, and electrical properties of a system's components. These interactions are a form of coupling dependent on magnitudes, rather than gradients, of the potentials. Examples are effects of temperature on magnitudes of viscosity and density of fluids and effects of pressure on permeability of porous media. In the second category, flows of heat, mass, and electrical charge can be driven by seemingly unrelated forces equal or related to gradients of the thermodynamic potentials. Examples are thermal diffusion in which a flow of mass is driven by a gradient of temperature and chemical osmosis in which a flow of volume is driven by a gradient of composition of a multicomponent fluid. These coupled transport processes can occur simultaneously with the direct processes described by the phenomenological laws of Fourier, Darcy, Fick, and $\mathrm{Ohm}$. This paper is concerned with the description of processes in the second category.

Laboratory investigations have shown that clay materials are particularly effective in supporting coupled flows. Thus, for example, it is reasonable to expect that low-permeability, argillaceous rocks could act as nonideal semipermeable membranes that allow flow of water but selectively retard movement of certain groundwater constituents.

The effects of coupled processes have been used to explain anomalous pressure and salinity data in argillaceous rocks such as clay and shale. BREDEHOEFr et al. (1963) discussed how the membrane properties of a layer of argillaceous rock could concentrate brines in subsurface formations. BERRY (1967) considered ultrafiltration (hyperfiltration) of hydrothermal solutions through clay to provide the best explanation of the distribution of chemical species in a thermal brine. GreEnBERg et al. (1973) used chemical osmosis in a model of coupled flows of salt and water in a coastal groundwater basin. MARINE AND FRITZ (1981) devised an osmotic model to account for anomalous pressures in a saline basin overlain by thick sediments. GRAF (1982) reviewed the roles of chemical osmosis and ultrafiltration in the origin of subsurface brines. In a somewhat different application, REED (1970) postulated thermal . osmotic and electrokinetic effects as causes of hydrologic phenomena observed near the cavity created by an underground nuclear explosion.

The membrane effects of clays and shales have been studied in the laboratory. Typically in these experiments, a disk of saturated clay or shale is placed between two chambers filled with either water or saline solutions. The flow of liquid through the sample is studied as a function of the gradients of temperature, pressure, concentration or electrical potential. Thus, MILNE et al. (1965) and LETEY AND KEMPER (1969) studied chemical osmosis and ultrafiltration between saline solutions separated by bentonite. YOUNG AND Low (1965) observed osmotic flow of a saline solution through natural siltstone and shale disks. OLSEN (1969) investigated electrokinetic phenomena associated with flows of liquid 
and charge through kaolinite. KHARAKA AND BERRY (1973) used a high pressure/high temperature filtration cell to study the membrane efficiencies of clays for several anions and monovalent and divalent cations usually present in subsurface water. SRIVASTAVA AND AVASTHI (1975) conducted an experiment to study thermal osmosis of water through kaolinite. In a series of papers, ElRICK et al. (1976), GroENeVelt and Elrick (1976), Groenevelt, Elrick and Blom (1976); and Groenevelt, Elrick and LARYEA (1976) presented experimental data on and a theoretical analysis of osmotic and electrokinetic coupled effects in montmorillonite. SRIVASTAVA AND ABRAHAM (1979) studied electro-osmosis of water through kaolinite and crysotile. Theoretical and experimental studies of fractionation of carbon and oxygen isotopes by ultrafiltration in clays have been reported by HAYDON AND GRAF (1986), PHILLIPS AND BENTLEY (1987), FRITZ et al. (1987), and DEMIR (1988). In general, experiments such as those mentioned above have been conducted in the steady state and have been limited to observations of effects of coupled flows on conditions in external reservoirs.

NEUziL (1986) recently published an important review paper on groundwater flow in low-permeability environments. His paper contains a wealth of references on qualitative studies in which coupled phenomena were used to explain anomalous pressures and subsurface brine formation and on laboratory experiments to study the membrane properties of argillaceous rocks. Though his article is concerned with topics other than coupled processes, Neuzil included a section on coupled flow in geologic membranes and noted several reasons why coupled phenomena have been largely ignored in analyzing flow in large low-permeability systems: (1) extrapolating small-scale laboratory experiments to large-scale flow systems is difficult, (2) excluding coupled effects makes the analysis less complicated and data requirements less demanding, and finally (3) "abundant experience with more permeable media, in which coupled flow is demonstrably unimportant, may have increased acceptance of the assumption of insignificant coupling" (NEUzIL, 1986, p. 1175). Also, NEUzIL pointed out that "important questions ... surround coupled flow behavior within the membrane medium itself, particularly when this behavior is transient" (NeUzIL, 1986, p. 1174) and that the external effects observed in laboratory experiments "may be less significant than those within low-permeability bodies with membrane properties" (NEUZIL, 1986, p. 1187) (emphasis added). NeUzIL stated his opinion that "the question of the significance of coupled flow in the subsurface has not been resolved" (NEUzIL, 1986, p. 1187).

The ability to correctly simulate transport by coupled processes is particularly important in lowpermeability environments. Neuzll $(1986$, p. 1163) has suggested that groundwater flow in these environments "appears to influence the evolution of certain hydrologic, geologic, and geochemical systems, may affect the accumulation of petroleum and ores, and probably has a role in the structural evolution of parts of the crust." MARINE AND FrITZ (1981) have discussed the importance of understanding the coupled processes of chemical osmosis and ultrafiltration in compacted clays separating fluids of different chemical composition. They noted that chemical potential may be a more significant driving force than either gravity or the pressure gradient, that without considering the effects of chemical osmosis and ultrafiltration the direction of water movement may be incorrectly deduced, and that the filtering effect of geologic media with membrane properties should be taken into account in geochemical analyses of the isotopic composition of groundwater.

Other situations where consideration of coupled flows may be important include waste disposal in low-permeability formations and the interaction of water and salt flows in soils. Low-permeability clay aquitards have been suggested as possible burial sites for solid, low-level radioactive wastes (FREEZE AND CHERRY, 1979, p. 452). Bentonite is being considered for use as a packing material around canisters of high-level radioactive wastes in an underground repository. In the latter case, large gradients of temperature, pressure and composition may cause flows of water and solute that cannot be predicted by the direct processes of advection and diffusion (CARNAHAN, 1984, 1985, 1986). Finally, the effects of coupled processes in soils having high salt contents may be significant because gradients of salt concentration can affect water migration in irrigated soils (LETEY AND KEMPER, 1969).

The goal of our work has been to develop a numerical simulator based on thermodynamically correct equations that describe heat and mass transport through a saturated porous medium by both direct and coupled processes. The simulator is intended to be a research tool for the study of transient heat and mass transport within a geologic membrane. Because of the difficulty of testing in low-permeability 
environments due to low flow rates and long response times, a numerical simulator can be used to gain insight into the relative significance of possible transport processes.

This paper, Part I of a set of two papers, provides the theoretical basis for development of a simulator of coupled processes. Although other approaches to the description of coupled processes are available [e.g., Hassanizadeh (1986a,b); Hassanizadeh and LeiJnse (1988)], we use a phenomenological formalism easily adaptable to analysis of experimental data. The thermodynamics of irreversible processes is reviewed briefly and then is used to formulate the governing equations for the thermodynamic variables temperature, pressure, and composition. The dissipation function is formulated and is used to develop the phenomenological equations in forms convenient for application to hydrogeologic problems involving chemical reactions. The phenomenological equations provide relations between the driving forces (gradients of the thermodynamic potentials) acting on the physico-chemical system and the fluxes of heat and matter appearing in the governing equations. The development in Part $I$ is general.

Part II (Jacobsen and CARnahan, 1990) of this set of papers describes a numerical method of solution of the governing equations posed with certain simplifying assumptions. As an application of the underlying theory and the numerical simulator, the effects of chemical osmosis, thermal filtration, thermal osmosis and ultrafiltration on heat and solute transport in the vicinity of a heat source buried in a saturated, clay-like material are considered.

\section{NOTATION}

$\tilde{A}_{f}$ thermodynamic affinity of $f$-th homogeneous chemical reaction, $\mathrm{J} / \mathrm{mol}$.

$\tilde{A}_{r}$ thermodynamic affinity of $r$-th heterogeneous chemical reaction, $\mathrm{J} / \mathrm{mol}$.

$c_{p, k} \quad$ specific heat capacity at constant pressure of phase $k, k \equiv f, j, n, J /(\mathrm{K} \mathrm{kg})$.

$c_{v, k}$ specific heat capacity at constant volume of phase $k, k \equiv f, j, n, J /(\mathrm{K} \mathrm{kg})$.

$C_{c}$ concentration of static energy, $\mathrm{J} / \mathrm{m}^{3}$.

$C_{i}, C_{j}$ concentration of mass of solute $i$ or solid phase $j, \mathrm{~kg} / \mathrm{m}^{3}$.

C. concentration of entropy, $\mathrm{J} /\left(\mathrm{K} \mathrm{m}^{3}\right)$.

$D_{i j}$ diffusion coefficient, $\mathrm{m}^{2} / \mathrm{s}$.

$E$ total static energy, J.

$f$ subscript denoting fluid phase.

$\mathcal{F}$ Faraday constant, $9.6487 \times 10^{4} \mathrm{C} / \mathrm{mol}$.

$g$ acceleration due to gravity, $9.80 \mathrm{~m} / \mathrm{s}^{2}$.

$h$ hydraulic head, $m$.

$h_{j} \quad$ specific enthalpy of solid phase $j, \mathrm{~J} / \mathrm{kg}$.

$\bar{H}_{i} \quad$ partial specific enthalpy of solute $i, \mathrm{~J} / \mathrm{kg}$.

$\vec{I}$ flux of electric charge, $\mathrm{A} / \mathrm{m}^{2}$.

$j$ subscript denoting reactive solid phase.

$J_{f}$ rate of $f$-th homogeneous chemical reaction, $\mathrm{mol} /\left(\mathrm{m}^{3} \mathrm{~s}\right)$.

$J_{r}$ rate of $r$-th heterogeneous chemical reaction, $\mathrm{mol} /\left(\mathrm{m}^{\mathrm{s}} \mathrm{s}\right)$.

$\vec{J}_{e}$ flux of static energy, $W / \mathrm{m}^{2}$.

$\vec{J}_{i}$ flux of solute in laboratory frame, $\mathrm{kg} /\left(\mathrm{m}^{2} \mathrm{~s}\right)$.

$\vec{J}_{i}^{0}$ flux of solute in solvent frame, $\mathrm{kg} /\left(\mathrm{m}^{2} \mathrm{~s}\right)$.

$\overrightarrow{J_{q}}$ flux of heat, $\mathrm{W} / \mathrm{m}^{2}$.

$\vec{J}$ flux of entropy, $\mathrm{W} /\left(\mathrm{K} \mathrm{m}^{2}\right)$.

$\vec{J}_{v}$ flux of volume, $\mathrm{m}^{3} /\left(\mathrm{m}^{2} \mathrm{~s}\right)$.

$k$ permeability, $\mathbf{m}^{2}$.

$\mathrm{K}$ thermal conductivity, $\mathrm{W} /(\mathrm{K} \mathrm{m})$.

$L_{f k}, L_{\mathrm{rl}}$ coefficients of chemical reaction rates, $\mathrm{mol}^{2} /\left(\mathrm{J} \mathrm{m}^{3} \mathrm{~s}\right)$.

$L_{e e}, L_{e e}^{\prime}$ coefficient of electrical conduction (Ohm's law), A/(V m).

$\mathrm{L}_{e i}, \mathrm{~L}_{e i}^{\prime}$ coefficient of sedimentation current, $(\mathrm{A} \mathrm{kg}) /(\mathrm{J} \mathrm{m})$.

$\mathrm{L}_{e q}, \mathrm{~L}_{e q}^{\prime}$ coefficient of Seebeck effect, $\mathrm{A} / \mathrm{m}$. 
$L_{e v}, L_{e v}^{\prime}$ coefficient of streaming current, $(A \mathrm{~m}) / \mathrm{N}$.

$\mathrm{L}_{\mathrm{ie}}, \mathrm{L}_{i e}^{\prime}$

$L_{i i}, L_{i i}^{\prime}$

$L_{i j}, L_{i j}^{\prime}$

$L_{i q}, L_{i q}^{\prime}$

$L_{i v}, L_{i v}^{\prime}$

$L_{q e}, L_{q e}^{\prime}$

$L_{q i}, L_{q i}^{\prime}$

$L_{q q}, L_{q q}^{\prime}$

$L_{q v}, L_{q v}^{\prime}$

$L_{v e}, L_{v e}^{\prime}$

$L_{v i}, L_{v i}^{\prime}$

$L_{v q}, L_{v q}^{\prime}$

$L_{v v} ; L_{v v}^{\prime}$

$m_{i}, m_{j}$

$M_{i}$

$n$

$N_{f}$

$N_{r}$ coefficient of electrophoresis, $(\mathrm{A} \mathrm{kg}) /(\mathrm{J} \mathrm{m})$. coefficient of mass diffusion (Fick's law), $\mathrm{kg}^{2} /(\mathrm{J} \mathrm{ms})$. coefficient of coupled mass diffusion for $i \neq j, \mathrm{~kg}^{2} /(\mathrm{J} \mathrm{ms})$. coefficient of thermal diffusion (Sorêt effect), $\mathrm{kg} /(\mathrm{m} \mathrm{s})$. coefficient of ultrafiltration, $(\mathrm{kg} \mathrm{m}) /(\mathrm{N} \mathrm{s})$. coefficient of Peltier effect, $\mathrm{A} / \mathrm{m}$. coefficient of Dufour effect, $\mathrm{kg} /(\mathrm{ms})$. coefficient of heat conduction (Fourier's law), W/m. coefficient of thermal filtration, $\mathrm{m}^{2} / \mathrm{s}$. coefficient of electro-osmosis, $(\mathrm{A} \mathrm{m}) / \mathrm{N}$. coefficient of chemical osmosis, $\left(\mathrm{kg} \mathrm{m}^{2}\right) /\left(\mathrm{J}_{\mathrm{s}}\right)$. coefficient of thermal osmosis, $\mathrm{m}^{2} / \mathrm{s}$. coefficient of direct advection (Darcy's law), $\mathrm{m}^{4} /(\mathrm{N} \mathrm{s})$. mass of solute $i$ or solid phase $\boldsymbol{j}, \mathbf{k g}$. molecular weight of solute $i, \mathrm{~kg} / \mathrm{mol}$. subscript denoting nonreactive solid phase. number of solute species. number of reactive solid phases. pressure, $\mathrm{N} / \mathrm{m}^{2}$. sum of second, fourth, and fifth terms in governing equations for $T$ and $P, N /\left(m^{2} s\right)$. gas constant, $8.314 \mathrm{~J} /(\mathrm{K} \mathrm{mol})$. number of homogeneous chemical reactions. number of heterogeneous chemical reactions. specific entropy of solid phase $j, J /(K \mathrm{~kg})$. total entropy, J/K.

entropy of phase $k, k \equiv f, j, n, \mathrm{~J} / \mathrm{K}$.

partial specific entropy of solute $i, J /(K \mathbf{~ k g})$.

time, $s$.

temperature, $\mathbf{K}$.

specific volume of solid phase $j, \mathrm{~m}^{3} / \mathrm{kg}$.

volume of porous medium, $\mathrm{m}^{3}$.

partial specific volume of solute $i, \mathrm{~m}^{3} / \mathrm{kg}$.

elevation above an arbitrary datum, $m$.

signed units of elementary charge of solute $i$.

specific electric charge of solute $i, \mathrm{C} / \mathrm{kg}$.

coefficient of thermal expansion of phase $k, k \equiv f, j, n, \mathrm{~K}^{-1}$.

coefficient in entropy balance and T-governing equations, $\mathrm{N} /\left(\mathrm{K} \mathrm{m}^{2}\right)$.

coefficient in entropy balance equation, $\mathrm{N} /\left(\mathrm{K} \mathrm{m}^{2}\right)$.

volume fraction of phase $k, k \equiv f, j, n$.

coefficient of isothermal compressibility of phase $k, k \equiv f, j, n, \mathrm{~m}^{2} / \mathrm{N}$.

coefficient in entropy balance and P-governing equations.

coefficient in entropy balance equation.

dynamic viscosity of fluid phase, $(\mathrm{N} \mathrm{s}) / \mathrm{m}^{2}$.

chemical potential of solute or reactive solid $i, \mathrm{~J} / \mathrm{m}^{3}$.

composition-dependent part of chemical potential of solute $i, \mathrm{~J} / \mathrm{m}^{3}$. stoichiometric coefficient of solute $i$ in reaction $k, k \equiv f, r$.

stoichiometric coefficient of solid $j$ in reaction $r$.

density of electric charge, $\mathrm{C} / \mathrm{m}^{3}$.

density of phase $k, k \equiv f, j, n, \mathrm{~kg} / \mathrm{m}^{3}$.

rate of production of entropy, $W /\left(\mathrm{K} \mathrm{m}^{3}\right)$.

specific potential energy of solute $i$ or reactive solid $j, \mathrm{~J} / \mathrm{m}^{3}$.

rate of dissipation of free energy, $W / \mathrm{m}^{3}$.

$\psi$ electrical potential, $\mathrm{V}$. 


\section{THE THERMODYNAMICS OF IRREVERSIBLE PROCESSES}

The governing equations discussed in the next section were derived using the thermodynamics of irreversible processes (TTIP). The fundamental ideas of TTIP were suggested by experiments performed in the 19th century (MILLER, 1960). The present form of the theory grew out of original work by ONSAGER (1931), who used statistical mechanics and fluctuation theory to formulate the basis of the present macroscopic theory. A brief history of the development of TTIP is given by DE GROOT AND MazUR (1969, Chap. 1). Extensive discussions of TTIP and its applications have been given by FitTs (1962), Katchalsky and Curran (1967) and DE Groot and Mazur (1969). Miller (1956) discussed the relation between classical thermodynamics and TTIP. GROENEVELT AND BOLT (1969) considered application of TTIP to irreversible processes in porous media.

TTIP describes nonequilibrium systems using the variables and state functions of classical (equilibrium) thermodynamics. TTIP is not, however, an extension of classical thermodynamics, but rather classical thermodynamics can be considered a subset of TTIP. This is evident from the following considerations. Any system can be characterized by the rate, $\sigma$, at which entropy is produced within the system by irreversible processes. TTIP, within the limits of its validity, includes all systems with $\sigma \geq 0$; classical thermodynamics considers only systems with $\sigma=0$ (Katchalsky aND CURRAN, 1967, Chapter 16).

The conceptual framework of TTIP is founded on three fundamental postulates. The remainder of this section is devoted to brief expositions of the postulates and their consequences.

TTIP invokes the first postulate in order to use the state functions of classical thermodynamics. The postulate states that that the thermodynamic quantities for a nonequilibrium system are the same functions of the local state variables as the corresponding equilibrium thermodynamic quantities (FITTs, 1962, p. 21). The validity of this postulate has been discussed by FitTs (1962, pp. 22-24). The postulate allows formulation of the balance equation of entropy for a system undergoing irreversible processes of change. This equation, the local formulation of the second law of thermodynamics, plays a central role in TTIP. It expresses the fact that the entropy of a volume element changes with time because entropy flows into the element and entropy is produced by irreversible processes within the element.

To relate the local rate of production of entropy, $\sigma$, to the irreversible processes creating entropy, the thermodynamic Gibbs equation (expressed as a differential equation in time) is used to connect the entropy balance equation to macroscopic conservation equations for mass, momentum, and energy. The conservation equations are expressed in differential (local) form involving derivatives with respect to space and time, degrees of freedom not defined in classical thermodynamics. This procedure leads to the result that the rate of entropy production, $\sigma$, in a system undergoing irreversible processes can be expressed as the sum of a series of products of the fluxes, $J_{i}$, and "forces", $X_{i}$, of the system:

$$
T \sigma=\sum_{i} J_{i} X_{i}
$$

where $T$ is the temperature. The fluxes are the flows of mass, momentum, and energy and the rates of chemical reactions appearing in the conservation laws. The forces are related either to the non-uniformity of the system (e.g., the gradient of temperature) or to the deviations of internal state variables from their equilibrium values (e.g., the affinity of a chemical reaction, formed by summing algebraically the chemical potentials of reactants and products). The term "force" is used somewhat loosely in TTIP, in that the forces are not necessarily defined in the strict sense of the (negative) gradient of a potential. When the fluxes and forces satisfy (1), a flux and a force with the same index $i$ are said to be conjugated. The product, $T \sigma$, is also known as the dissipation function and in this work will be denoted henceforth by $\Phi$.

The second postulate of TTIP states that each flux in the system can be written as a linear combination of all of the forces:

$$
J_{i}=\sum_{j} L_{i j} X_{j}
$$

These equations are called the phenomenological equations, and the coefficients, $L_{i j}$, are the phenomeno- 
logical coefficients. For the processes studied in this work, the phenomenological coefficients are either scalars or tensors of rank two. The relations in (2) extend the application of linear phenomenological laws, which include the well-known Fourier's law, Darcy's law, Fick's law and Ohm's law, to systems in which there are interacting flows. The coupling of the fluxes and forces represented by the phenomenological equations is essentially what is meant by "thermodynamic" coupling: fluxes of heat and matter may be caused by seemingly unrelated (nonconjugated) forces. For example, in thermal diffusion, also known as the Sorêt effect, a gradient of temperature gives rise to a mass flux. In the diffusive-thermal (Dufour) effect, a gradient of solute chemical potential induces a heat flux. KATCHALSKY AND CURRAN (1967, $\$ 8.2)$ have discussed the validity of the linear relations in general. For a given system, the validity of (2) is subject to experimental confirmation.

Restrictions on (2) exist for systems having certain elements of symmetry. Curie's theorem states that fluxes and forces differing by an odd integer in tensorial rank cannot interact in isotropic systems (FITTs, 1962, pp. 35-36; KATCHALSKY AND CURRAN, 1967, \$8.3). In particular, the affinity of a chemical reaction, a scalar, cannot give rise to any vector flux. Conversely, vector forces (i.e., gradients of temperature, pressure, or compositional variables) cannot couple to rates of chemical reactions. These restrictions can be extended to systems with symmetries lower than isotropic (CARNARAN, 1976).

We note that, in general, the phenomenological coefficients can be functions of temperature, pressure, and composition.

The third postulate of TTIP states that the phenomenological coefficients in (2) satisfy the symmetry relations,

$$
L_{i j}=L_{j i}
$$

if the fluxes and forces satisfy certain conditions. The relations in (3) are known as the Onsager reciprocal relations and were originally derived by ONSAGER (1931) from considerations of microscopic reversibility. A consequence of (3) is that coupled effects must occur in pairs, if they occur at all. The validity of the reciprocal relations for a given system is also subject to experimental confirmation. The conditions for validity of the reciprocal relations have been the subject of intense debate [cf. CoLEMAN AND TRUESDELL (1960); MASON (1974)] centering on the precise definitions of fluxes and forces. Many authors, among them KATCHALSKY AND CURRAN $(1967, \$ 8.4)$ and Miller $(1960$, p. 17), state that the phenomenological coefficients will satisfy the reciprocal relations if the fluxes in (1) are independent, if the linear laws in (2) are valid, and if the fluxes and forces in (2) are the same as those in (1). The more restrictive conditions on the fluxes and forces have been discussed by FITTs (1962, $\$ 4.3$ and Appendix B), ANDERSON AND Graf (1976, pp. 108-114), and Miller (1974, p. 189). In this paper, the fluxes and forces are chosen from a dissipation function to be derived in a later section. Following FirTs $(1962$, p. 39), we have assumed that the fluxes and forces satisfy the additional conditions required to guarantee the reciprocal relations.

The phenomenological equations and reciprocal relations have been verified for many physical systems. In an early paper, MILLER (1960) reviewed experiments measuring thermoelectric effects, electrokinetic effects, isothermal diffusion and anisotropic heat conduction and concluded that the "experimental evidence is overwhelmingly in favor of the Onsager reciprocal relations" (MILLER, 1960, p. 35). In a later paper, MILLER (1974) included data on chemical reactions, thermomagnetism and galvanomagnetism, and transference in electrolyte solutions. He came to the same conclusion (MILLER, 1974, p. 208) as before, but added the following disclaimer for chemical reactions: the linear laws are valid only for reactions very close to equilibrium (MILLER, 1974, p. 195). For chemical reactions close to equilibrium, KATCHALSKY AND CURRAN $(1967, \$ 88.5)$ have shown that the reciprocal relations are equivalent to the principle of detailed balance.

Several authors have investigated the phenomenological equations and reciprocal relations in claywater systems. LETEY AND KEMPER (1969) formulated linear laws describing the movement of water and salt through bentonite. They measured the phenomenological coefficients in the linear laws for their system and found confirmation, within experimental error, of the single Onsager reciprocal relation possible for their system. OLSEN (1969) measured the flows of liquid, charge, and electrolyte through 
saturated kaolinite. His experiments confirmed one of three possible Onsager reciprocal relations and the linearity of a set of phenomenological equations expressing the fluxes of volume, charge and salt in terms of gradients of hydraulic head, electrical potential, and electrolyte chemical potential. SRIVASTAVA AND AVASTHI (1975) performed experiments to study thermal osmosis through kaolinite. They expressed the fluxes of heat and water in terms of gradients of temperature and pressure. Their experiments confirmed their proposed linear laws over a large range of temperature gradients, but did not provide a test of the single reciprocal relation possible for their system. Srivastava aNd ABraham (1979) studied electroosmosis of water through composite clay membranes consisting of kaolinite and crysotile clay membrane elements arranged in series and in parallel. The linear phenomenological equations and the reciprocal relations were shown to be valid for both composite membrane arrangements and also for the component clay membrane elements.

\section{DERIVATION OF THE GOVERNING EQUATIONS}

In this section, equations describing the rates of change of composition, temperature and pressure in terms of the forces and fluxes in the system are derived. The equations of conservation of mass are stated first. To derive governing equations for temperature and pressure, we begin by emulating the approach used by FITTs (1962, pp. 51-53) and write two equations for entropy balance, one expressing the total differential entropy as a function of pressure, volume and composition and the other as a function of temperature, volume, and composition. After eliminating partial derivatives of entropy from these equations, we depart from FiTTs's approach and adopt the procedure used by KATCHALSKY AND CurRan (1967, Chapter 7) to replace extensive state variables by their intensive counterparts. The latter approach lends itself more naturally to treatment of open systems. We regard the system as a continuum and adopt the results of GROENEVELT AND BOLT (1969); specifically, we neglect energy transfer by viscous processes. The Gibbs equation and other thermodynamic relations are then used to eliminate entropy from the balance equations. Using energy and mass conservation equations, the resulting expressions are rewritten in terms of the intensive state variables and a set of independent fluxes. These new results extend the governing equations derived by FiTTs (1962, p. 53) to include open systems.

Governing equations are derived for a thermodynamically open system comprised of a saturated porous matrix with macroscopic porosity $\epsilon_{f}$. The fluid phase consists of $N_{f}$ solute species, denoted by index $i$, and a solvent, denoted by index 0 . The solid phase consists of $N_{r}$ reactive compounds, denoted by index $j$, and a nonreactive substrate, denoted by index $n$. Each reactive solid occupies fraction $\epsilon_{j}$, and the substrate fraction $\epsilon_{n}$, of the volume of the porous matrix. There are $R_{f}$ homogeneous reactions, denoted by index $f$, in the fluid phase and $R_{r}$ heterogeneous reactions, denoted by index $r$, between components of the fluid phase and the reactive solids. All vector fluxes (areal densities of flow of energy, matter, and electric charge) are referred to a unit area of the porous matrix. Rates of chemical reactions are expressed as moles per unit time per unit volume of porous matrix. Concentrations of solute species are referred to a unit volume of the fluid phase, but other concentrations (of solids, energy, entropy) are referred to a unit volume of the porous matrix. Mass density of a solid is referred to a unit volume of the solid.

\section{The Mass Conservation Equations}

The mass conservation equations are, for water and solutes,

$$
\frac{\partial}{\partial t}\left(\epsilon_{f} C_{i}\right)=-\vec{\nabla} \cdot \vec{J}_{i}+\sum_{f=1}^{R_{f}} M_{i} \nu_{i f} J_{f}+\sum_{r=1}^{R_{r}} M_{i} \nu_{i r} J_{r}, \quad i=0, \ldots, N_{f}
$$

and, for reactive solids,

$$
\frac{\partial C_{j}}{\partial t}=\sum_{r=1}^{R_{r}} M_{j} \nu_{j r} J_{r}, \quad j=1, \ldots, N_{r}
$$


where $C_{i}$ is the concentration of solution species $i, C_{j}$ is the concentration of reactive solid $j, \vec{J}_{i}$ is the mass flux of solution species $i$ in a coordinate system fixed with respect to the porous matrix (the "laboratory. reference frame"), $J_{f}$ is the rate of the $f$-th homogeneous reaction among solution species, $J_{r}$ is the rate of the $r$-th heterogeneous reaction between solution species and a reactive solid, $M_{i}$ is the molecular weight of solute $i, M_{j}$ is the molecular weight of solid $j, \nu_{i f}$ are the stoichiometric coefficients for the homogeneous reactions, and $\nu_{i r}$ and $\nu_{j r}$ are the stoichiometric coefficients for the heterogeneous reactions. Stoichiometric coefficients are negative for reactants, positive for products and zero for nonreacting species or compounds. For brevity, the notation below will be used throughout the remaining derivation:

$$
\begin{aligned}
A_{i f} & =\sum_{f=1}^{R_{f}} M_{i} \nu_{i f} J_{f}, \quad i=0, \ldots, N_{f}, \\
A_{i r} & =\sum_{r=1}^{R_{r}} M_{i} \nu_{i r} J_{r}, \quad i=0, \ldots, N_{f}, \\
A_{j r} & =\sum_{r=1}^{R_{r}} M_{j} \nu_{j r} J_{r}, \quad j=1, \ldots, N_{r} .
\end{aligned}
$$

The rate of change of the volume fraction of solid $j, \epsilon_{j}$, is given by

$$
\frac{\partial \epsilon_{j}}{\partial t}=v_{j} \frac{\partial C_{j}}{\partial t}
$$

where $v_{j}$ is the specific volume of solid $j$. Then, using

$$
\epsilon_{f}+\sum_{j=1}^{N_{r}} \epsilon_{j}+\epsilon_{n}=1
$$

and keeping $\epsilon_{n}$ constant gives

$$
\frac{\partial \epsilon_{f}}{\partial t}=-\sum_{j=1}^{N_{r}} \frac{\partial \epsilon_{j}}{\partial t}=-\sum_{j=1}^{N_{r}} v_{j} \frac{\partial C_{j}}{\partial t}
$$

or, using (4b) and (5c),

$$
\begin{aligned}
& \frac{\partial \epsilon_{j}}{\partial t}=v_{j} A_{j r} \\
& \frac{\partial \epsilon_{f}}{\partial t}=-\sum_{j=1}^{N_{r}} v_{j} A_{j r}
\end{aligned}
$$

\section{The Entropy Balance Equations}

The total entropy, $S$, in a volume, $V$, of saturated porous matrix is composed of contributions from each phase, fluid and solid. If $S_{f}, S_{j}$ for $j=1, \ldots, N_{r}$, and $S_{n}$ are the entropies of each of the fluid, 
reactive solid, and nonreactive solid phases in volume $V$; the total differential of the entropy, $S$, is given by

$$
d S=d S_{f}+\sum_{j=1}^{N_{r}} d S_{j}+d S_{n}
$$

Entropy may be regarded as a function of temperature, volume and composition, or as a function of pressure, volume and composition. Following FitTs (1962, p. 51), we write expressions for total differentials of the entropies of each phase according to each of the two fundamental representations. The result is two sets of three equations, each set of equations relating the total differentials of the entropies of the three phases to the partial derivatives of each phase's entropy with respect to one set of thermodynamic variables. The partial derivatives are eliminated by expressions relating each of them to partial specific entropies of fluid components, specific entropies of solids, and several material coefficients. Each revised set of differential phase entropies is substituted separately into (10), giving the two equations

$$
\begin{aligned}
& d S=\Gamma_{1} \frac{V}{T} d T+\Gamma_{2} d V+\sum_{i=0}^{N_{f}}\left(\bar{S}_{i}-\frac{\beta_{f}}{\kappa_{f}} \nabla_{i}\right) d m_{i}+\sum_{j=1}^{N_{r}}\left(s_{j}-\frac{\beta_{j}}{\kappa_{j}} v_{j}\right) d m_{j} \\
& d S=\Lambda_{1} \frac{V}{T} d P+\frac{\Lambda_{2}}{T} d V+\sum_{i=0}^{N_{f}}\left(\bar{S}_{i}-\frac{\rho_{f} c_{p, f}}{\beta_{f} T} \bar{V}_{i}\right) d m_{i}+\sum_{j=1}^{N_{r}}\left(\delta_{j}-\frac{c_{p, j}}{\beta_{j} T}\right) d m_{j}
\end{aligned}
$$

with

$$
\begin{aligned}
& \Gamma_{1}=\epsilon_{f} \rho_{f} c_{v, j}+\sum_{j=1}^{N_{r}} \epsilon_{j} \rho_{j} c_{v j}+\epsilon_{n} \rho_{n} c_{v, n} \\
& \Gamma_{2}=\epsilon_{f} \frac{\beta_{f}}{\kappa_{j}}+\sum_{j=1}^{N_{r}} \epsilon_{j} \frac{\beta_{j}}{\kappa_{j}}+\epsilon_{n} \frac{\beta_{n}}{\kappa_{n}}, \\
& \Lambda_{1}=\epsilon_{f} \rho_{f} c_{v, j} \frac{\kappa_{f}}{\beta_{f}}+\sum_{j=1}^{N_{r}} \epsilon_{j} \rho_{j} c_{v, j} \frac{\kappa_{j}}{\beta_{j}}+\epsilon_{n} \rho_{n} c_{v, n} \frac{\kappa_{n}}{\beta_{n}}, \\
& \Lambda_{2}=\frac{\epsilon_{f} \rho_{f} c_{p, f}}{\beta_{f}}+\sum_{j=1}^{N_{r}} \frac{\epsilon_{j} \rho_{j} c_{p, j}}{\beta_{j}}+\frac{\epsilon_{n} \rho_{n} c_{p, n}}{\beta_{n}},
\end{aligned}
$$

where $T$ is temperature, $P$ is pressure, $m_{0}$ is the mass of the solvent, $m_{i}$ is the mass of solute $i, m_{j}$ is the mass of reactive solid $j, \bar{S}_{i}$ is the partial specific entropy and $\nabla_{i}$ the partial specific volume of solute $i, s_{j}$ is the specific entropy and $v_{j}$ the specific volume of solid $j$, and for phase $k$ ( $k$ representing $f, j$ or $n$ ), $c_{v, k}$ is the specific heat capacity at constant volume, $c_{p, k}$ is the specific heat capacity at constant pressure, $\beta_{k}$ is the coefficient of thermal expansion $\left[\beta_{k}=(1 / V)(\partial V / \partial T)_{P, m}\right], \kappa_{k}$ is the coefficient of isothermal compressibility $\left[\kappa_{k}=-(1 / V)(\partial V / \partial P)_{T, m}\right]$, and $\rho_{k}$ is the mass density of the phase.

Details of the derivation of (11) and (12) are given in Appendix A.

\section{Balance Equations for Intensive State Variables}

We next replace the extensive variables, $S, m_{i}, i=0, \ldots, N_{f}$, and $m_{j}, j=1, \ldots, N_{r}$, in (11) and (12) by their intensive counterparts and eliminate the extensive variable, $V$. We follow the procedure of KatchalsKY AND CURRAN (1967, Chapter 7) and use the following relations,

$$
S=V C_{s}
$$




$$
\begin{aligned}
& m_{i}=\epsilon_{f} V C_{i}, \quad i=0, \ldots, N_{f}, \\
& m_{j}=V C_{j}, \quad j=1, \ldots, N_{r},
\end{aligned}
$$

where $C_{a}$ is the concentration of entropy in the saturated porous matrix. The relations (14) are substituted into (11) and (12). The results of the substitution are integrated, and the integral forms are used to eliminate terms multiplying the differential of volume, $d V$, in the expansions of the substituted differential forms. Details of these operations are given in Appendix B. The time derivatives of the remaining terms are formed and the results are, after multiplying by $T$,

$$
\begin{aligned}
& \Gamma_{1} \frac{\partial T}{\partial t}=T \frac{\partial C_{s}}{\partial t}-\sum_{i=0}^{N_{j}}\left(T \bar{S}_{i}-\frac{\beta_{f} T}{\kappa_{f}} \bar{V}_{i}\right) \frac{\partial}{\partial t}\left(\epsilon_{f} C_{i}\right)-\sum_{j=1}^{N_{r}}\left(T s_{j}-\frac{\beta_{j} T}{\kappa_{j}} v_{j}\right) \frac{\partial C_{j}}{\partial t} \\
& \Lambda_{1} \frac{\partial P}{\partial t}=T \frac{\partial C_{s}}{\partial t}-\sum_{i=0}^{N_{f}}\left(T \bar{S}_{i}-\frac{\rho_{f} c_{p_{1} f}}{\beta_{f}} \nabla_{i}\right) \frac{\partial}{\partial t}\left(\epsilon_{f} C_{i}\right)-\sum_{j=1}^{N_{r}}\left(T s_{j}-\frac{c_{p, j}}{\beta_{j}}\right) \frac{\partial C_{j}}{\partial t}
\end{aligned}
$$

The left-hand sides of (15) and (16) contain the desired time derivatives of $T$ and $P$. To further the development of the governing equations, the time derivatives must be eliminated from the right-hand sides of (15) and (16). This is done using the Gibbs equation and conservation equations for mass and energy.

\section{Introduction of the Gibbs Equation and Other Conservation Equations}

The Gibbs equation is used to eliminate the time derivative of $C$, on the right-hand sides of (15) and (16). As shown in Appendix C, the Gibbs equation is used to derive the following partial differential equation for a volume element of the porous medium:

$$
T \frac{\partial C_{s}}{\partial t}=\frac{\partial C_{e}}{\partial t}-\sum_{i=0}^{N_{f}}\left(\mu_{i}+\varphi_{i}\right) \frac{\partial}{\partial t}\left(\epsilon_{f} C_{i}\right)-\sum_{j=1}^{N_{F}}\left(\mu_{j}+\varphi_{j}\right) \frac{\partial C_{j}}{\partial t}
$$

where $C_{e}$ is the concentration of static energy, $\mu_{i}$ and $\varphi_{i}$ are the chemical potential and specific potential energy of solute $i$, and $\mu_{j}$ and $\varphi_{j}$ are the chemical potential and specific potential energy of reactive solid $j$. The partial derivative of $C_{e}$ on the right-hand side of (17) is eliminated using the conservation equation for static energy (Groenevelt aNd Bolt, 1969),

$$
\frac{\partial C_{e}}{\partial t}=-\vec{\nabla} \cdot \vec{J}_{e}
$$

where $\vec{J}_{e}$ is the flux of static energy. Here we neglect conversion of kinetic into static energy by viscous processes. The static energy flux $\vec{J}_{e}$ is composed of the conductive heat flux, $\vec{J}_{q}$, and fluxes of sensible heat and potential energy carried by each component of the fluid phase (GROENEVELT AND BoLT, 1969):

$$
\vec{J}_{e}=\vec{J}_{q}+\sum_{i=0}^{N_{f}} \vec{J}_{i}\left(\vec{H}_{i}+\varphi_{i}\right)
$$

where $\bar{H}_{i}$ is the partial specific enthalpy of solute $i$. As shown in Appendix C, the time derivatives on the right-hand sides of (15) and (16) now can be eliminated by use of (17)-(19) and the mass conservation 
equations, (4). After substitutions for $\mu_{i}$ and $\mu_{j}$, the results are:

$$
\begin{aligned}
\Gamma_{1} \frac{\partial T}{\partial t}= & -\vec{\nabla} \cdot \vec{J}_{q}-\sum_{i=0}^{N_{f}}\left[\vec{J}_{i} \cdot \vec{\nabla}\left(H_{i}+\varphi_{i}\right)+\frac{\beta_{f} T}{\kappa_{f}} \nabla_{i}\left(\vec{\nabla} \cdot \vec{J}_{i}\right)\right] \\
& -\sum_{i=0}^{N_{f}}\left(\bar{H}_{i}+\varphi_{i}-\frac{\beta_{f} T}{\kappa_{f}} \nabla_{i}\right)\left(A_{i f}+A_{i r}\right)-\sum_{j=1}^{N_{r}}\left(h_{j}+\varphi_{j}-\frac{\beta_{j} T}{\kappa_{j}} v_{j}\right) A_{j r} \\
\Lambda_{1} \frac{\partial P}{\partial t}= & -\vec{\nabla} \cdot \vec{J}_{q}-\sum_{i=0}^{N_{f}}\left[\vec{J}_{i} \cdot \vec{\nabla}\left(\bar{H}_{i}+\varphi_{i}\right)+\frac{\rho_{f} c_{p, f}}{\beta_{f}} \bar{V}_{i}\left(\vec{\nabla} \cdot \vec{J}_{i}\right)\right] \\
& -\sum_{i=0}^{N_{f}}\left(\bar{H}_{i}+\varphi_{i}-\frac{\rho_{f} c_{p, f}}{\beta_{f}} \nabla_{i}\right)\left(A_{i f}+A_{i r}\right)-\sum_{j=1}^{N_{r}}\left(h_{j}+\varphi_{j}-\frac{c_{p, j}}{\beta_{j}}\right) A_{j r}
\end{aligned}
$$

Equations (20) and (21) are very nearly the final forms of the governing equations. The major step remaining is to replace the laboratory-referenced mass fluxes, $\vec{J}_{i}$, by the volume flux, $\vec{J}_{v}$, and a set of modified solute mass fluxes. As shown in the subsequent section on the phenomenological equations, the phenomenological equation for $\vec{J}_{v}$ will then contain explicitly an analog of Darcy's law as one contribution to the volume flux (specific discharge). The forms of the governing equations incorporating the volume flux and the modified solute mass fluxes are the most useful forms for analysis of transport processes in multicomponent, subsurface fluids.

\section{Introduction of the Volume Flux and Diffusional Solute Fluxes}

The volume flux, the solvent flux and the solute fluxes in the laboratory reference frame do not form a set of independent fluxes because the volume flux is defined by

$$
\vec{J}_{v}=\sum_{i=0}^{N_{f}} \bar{V}_{i} \vec{J}_{i}
$$

Therefore, in order to introduce the volume flux into the governing equations, the solvent flux will be eliminated from them. In the process of doing this, a set of independent solute fluxes will be introduced.

The only terms in (20) and (21) that involve the solvent and solute fluxes are the first sums on the right-hand sides of each equation. For simplicity, each sum will be represented by

$$
\sum_{i=0}^{N_{f}}\left[\vec{J}_{i} \cdot \vec{\nabla}\left(\vec{H}_{i}+\varphi_{i}\right)+\chi \bar{V}_{i} \vec{\nabla} \cdot \vec{J}_{i}\right]
$$

where

$$
\chi=\frac{\beta_{f} T}{\kappa_{f}}
$$

in the case of $(20)$ and

$$
\chi=\frac{\rho_{f} c_{p, f}}{\beta_{f}}
$$

in the case of (21). The term $\vec{\nabla}\left(\bar{H}_{i}+\varphi_{i}\right)$ in expression (23) is rewritten as follows. Expanding the 
gradient of chemical potential of solute $i$ gives (KATCHALSKY AND CURRAN, 1967, p. 83)

$$
\vec{\nabla} \mu_{i}=-\bar{S}_{i} \vec{\nabla} T+\bar{V}_{i} \vec{\nabla} P+\vec{\nabla} \mu_{i}^{c}
$$

where $\mu_{i}^{e}$ is the composition-dependent part of the chemical potential. Using (C-7a) and (26), $\vec{\nabla}\left(\bar{H}_{i}+\varphi_{i}\right)$ is written

$$
\vec{\nabla}\left(\mathscr{H}_{i}+\varphi_{i}\right)=\bar{\nabla}_{i} \vec{\nabla} P+\vec{\nabla} \varphi_{i}+\vec{\nabla} \mu_{i}^{e}+T \vec{\nabla} \bar{S}_{i}
$$

The Gibbs-Duhem equation for the composition-dependent part of the chemical potential is used to express $\mu_{0}^{e}$ in terms of the $\mu_{i}^{e}$ (KATCHALSKY AND CURRAN, 1967, p. 54):

$$
\vec{\nabla} \mu_{0}^{c}=-\sum_{i=1}^{N_{f}} \frac{C_{i}}{C_{0}} \vec{\nabla} \mu_{i}^{e}
$$

Forming the dot products of $\vec{J}_{i}$ and each side of (27), summing over $i$ from 0 to $N_{f}$, and substituting (28) into the result yield

$$
\sum_{i=0}^{N_{j}} \vec{J}_{i} \cdot \vec{\nabla}\left(\bar{H}_{i}+\varphi_{i}\right)=\sum_{i=0}^{N_{j}} \vec{J}_{i} \cdot\left(\nabla_{i} \vec{\nabla} P+T \vec{\nabla} \bar{S}_{i}+\vec{\nabla} \varphi_{i}\right)+\sum_{i=1}^{N_{f}}\left(\vec{J}_{i}-\frac{C_{i}}{C_{0}} J_{0}\right) \cdot \vec{\nabla} \mu_{i}^{c}
$$

The definition of the modified solute fluxes is now introduced: the solute fluxes referred to the motion of the solvent are defined by

$$
\vec{J}_{i}^{\circ}=\vec{J}_{i}-\frac{C_{i}}{C_{0}} \vec{J}_{0}, \quad i=1, \ldots, N_{f}
$$

The fluxes $\vec{J}_{i}^{0}$ are referred to as the solvent-fixed fluxes or diffusional fluxes of solutes (KATCHALSKY AND Curran, 1967, Chapter 9).

Using (22), (30) and the relation

$$
\sum_{i=0}^{N_{f}} C_{i} \bar{V}_{i}=1
$$

the fluxes, $\vec{J}_{i}, i=0, \ldots, N_{f}$, are written in terms of the volume flux and the diffusional solute fluxes:

$$
\begin{aligned}
& \vec{J}_{0}=C_{0}\left(\vec{J}_{v}-\sum_{j=1}^{N_{f}} \bar{V}_{j} \vec{J}_{j}^{0}\right), \\
& \vec{J}_{i}=\vec{J}_{i}^{0}+C_{i}\left(\vec{J}_{v}-\sum_{j=1}^{N_{f}} \bar{V}_{j} \vec{J}_{j}^{0}\right), \quad i=1, \ldots, N_{\rho} .
\end{aligned}
$$

Using (30) in (29) and inserting the result into (23) lead to 


$$
\begin{aligned}
\sum_{i=0}^{N_{g}}\left[\vec{J}_{i} \cdot \vec{\nabla}\left(H_{i}+\varphi_{i}\right)+\chi \nabla_{i} \vec{\nabla} \cdot \vec{J}_{i}\right] & =\sum_{i=0}^{N_{f}} \vec{J}_{i} \cdot\left(\vec{\nabla}_{i} \vec{\nabla} P+T \vec{\nabla} \bar{S}_{i}+\vec{\nabla} \varphi_{i}\right) \\
& +\sum_{i=1}^{N_{f}} \vec{J}_{i}^{0} \cdot \vec{\nabla}_{i}^{e}+\chi \sum_{i=0}^{N_{f}} \nabla_{i} \vec{\nabla} \cdot \vec{J}_{i} .
\end{aligned}
$$

Substituting (32) and (33) into (34) and using the relation

$$
\chi \sum_{i=0}^{N_{f}} \nabla_{i} \vec{\nabla} \cdot \vec{J}_{i}=\chi \vec{\nabla} \cdot \vec{J}_{v}-\sum_{i=0}^{N_{f}} \vec{J}_{i} \cdot \chi \vec{\nabla} \nabla_{i}
$$

yield

$$
\begin{aligned}
& \sum_{i=0}^{N_{f}}\left[\vec{J}_{i} \cdot \vec{\nabla}\left(\bar{H}_{i}+\varphi_{i}\right)+\chi \nabla_{i} \vec{\nabla} \cdot \vec{J}_{i}\right]=\sum_{i=1}^{N_{f}} \vec{J}_{i}^{\circ} \cdot\left(\vec{\nabla} \mu_{i}^{c}+\vec{\nabla} \varphi_{i}+T \vec{\nabla} \bar{S}_{i}-\chi \vec{\nabla} \nabla_{i}\right) \\
& \quad+\left(\vec{J}_{v}-\sum_{j=1}^{N_{f}} \bar{V}_{j} \vec{J}_{j}^{\circ}\right) \cdot \sum_{i=0}^{N_{f}} C_{i}\left(\vec{\nabla} \varphi_{i}+T \vec{\nabla} \bar{S}_{i}-\chi \vec{\nabla} \bar{V}_{i}\right)+\vec{J}_{v} \cdot \vec{\nabla} P+\chi \vec{\nabla} \cdot \vec{J}_{v}
\end{aligned}
$$

Substituting (36) into (20) and (21) gives the most general forms of the governing equations for temperature and pressure:

$$
\begin{aligned}
& \Gamma_{1} \frac{\partial T}{\partial t}=-\vec{\nabla} \cdot \vec{J}_{q}-\vec{J}_{v} \cdot \vec{\nabla} P-\frac{\beta_{f} T}{\kappa_{f}}\left(\vec{\nabla} \cdot \vec{J}_{v}\right)-\sum_{i=1}^{N_{f}} \vec{J}_{i}^{0} \cdot\left(\vec{\nabla} \mu_{i}^{e}+\vec{\nabla} \varphi_{i}+T \vec{\nabla} \bar{S}_{i}-\frac{\beta_{f} T}{\kappa_{f}} \vec{\nabla} \nabla_{i}\right) \\
& -\left(\vec{J}_{v}-\sum_{j=1}^{N_{f}} \nabla_{j} \vec{J}_{j}^{0}\right) \cdot \sum_{i=0}^{N_{f}} C_{i}\left(\vec{\nabla} \varphi_{i}+T \vec{\nabla} \bar{S}_{i}-\frac{\beta_{j} T}{\kappa_{f}} \vec{\nabla} \nabla_{i}\right) \\
& -\sum_{i=0}^{N_{f}}\left(\bar{H}_{i}+\varphi_{i}-\frac{\beta_{j} T}{\kappa_{f}} \bar{V}_{i}\right)\left(A_{i j}+A_{i r}\right)-\sum_{j=1}^{N_{r}}\left(h_{j}+\varphi_{j}-\frac{\beta_{j} T}{\kappa_{j}} v_{j}\right) A_{j r}, \\
& \Lambda_{1} \frac{\partial P}{\partial t}=-\vec{\nabla} \cdot \vec{J}_{q}-\vec{J}_{v} \cdot \vec{\nabla} P-\frac{\rho_{f} c_{p, f}}{\beta_{f}}\left(\vec{\nabla} \cdot \vec{J}_{v}\right)-\sum_{i=1}^{N_{f}} \vec{J}_{i}^{0} \cdot\left(\vec{\nabla} \mu_{i}^{e}+\vec{\nabla} \varphi_{i}+T \vec{\nabla} \bar{S}_{i}-\frac{\rho_{f} c_{p, f}}{\beta_{f}} \vec{\nabla} \bar{V}_{i}\right) \\
& -\left(\vec{J}_{v}-\sum_{j=1}^{N_{f}} \bar{V}_{j} \vec{J}_{j}^{0}\right) \cdot \sum_{i=0}^{N_{j}} C_{i}\left(\vec{\nabla} \varphi_{i}+T \vec{\nabla} \bar{S}_{i}-\frac{\rho j c_{p, f}}{\beta_{f}} \vec{\nabla} \bar{V}_{i}\right) \\
& -\sum_{i=0}^{N_{j}}\left(\bar{H}_{i}+\varphi_{i}-\frac{\rho j c_{p j}}{\beta_{f}} \bar{V}_{i}\right)\left(A_{i f}+A_{i r}\right)-\sum_{j=1}^{N_{r}}\left(h_{j}+\varphi_{j}-\frac{c_{p j}}{\beta_{j}}\right) A_{j r}
\end{aligned}
$$




\section{The Governing Equations for a Particular Potential, $\varphi_{i}$}

In many applications, the specific potential energy, $\varphi_{i}$, of a solute species, $i$, is given by

$$
\varphi_{i}=g z+Z_{i} \psi
$$

where $g$ is the acceleration due to gravity, $z$ is the local elevation relative to an arbitrary datum, $\psi$ is an electrical potential, and $Z_{i}$, the specific electric charge of species $i$, is defined by

$$
Z_{i}=\frac{z_{i} \mathcal{F}}{M_{i}}
$$

where $M_{i}$ and $z_{i}$ are the molecular weight and the electric charge of solute $i$, respectively, and $\mathcal{F}$ is the Faraday constant. We now examine the forms taken by (37) and (38) when the potential, $\varphi_{i}$, assumes the form of (39).

For convenience, we define a quantity, $Q$, equal to the sum of the second, fourth, and fifth terms on the right-hand sides of (37) and (38):

$$
\begin{aligned}
Q= & -\vec{J}_{v} \cdot \vec{\nabla} P-\sum_{i=1}^{N_{j}} \vec{J}_{i}^{\circ} \cdot\left(\vec{\nabla} \mu_{i}^{e}+\vec{\nabla} \varphi_{i}+T \vec{\nabla} \bar{S}_{i}-\chi \vec{\nabla} \nabla_{i}\right) \\
& -\left(\vec{J}_{v}-\sum_{j=1}^{N_{f}} \bar{V}_{j} \vec{J}_{j}^{\circ}\right) \cdot \sum_{i=0}^{N_{f}} C_{i}\left(\vec{\nabla} \varphi_{i}+T \vec{\nabla} \bar{S}_{i}-\chi \vec{\nabla} \bar{V}_{i}\right),
\end{aligned}
$$

where $\chi$ has the same meanings as in (24) and (25). The fluid mass density, $\rho_{f}$, is defined by

$$
\rho_{f}=\sum_{i=0}^{N_{f}} C_{i},
$$

and the local density of electric charge, $\rho_{c}$, is defined by

$$
\rho_{c}=\sum_{i=1}^{N_{j}} Z_{i} C_{i},
$$

assuming that $Z_{0}$, the specific electric charge of the solvent, is zero. Then, substituting (39) into (41) and using (42) and (43) give

$$
\begin{aligned}
Q= & -\vec{J}_{v} \cdot\left(\vec{\nabla} P+\rho_{f} g \vec{\nabla} z+\rho_{c} \vec{\nabla} \psi\right) \\
& -\sum_{i=1}^{N_{f}} \vec{J}_{i}^{0} \cdot\left[\vec{\nabla} \mu_{i}^{e}+\left(1-\rho_{j} \bar{V}_{i}\right) g \vec{\nabla} z+\left(Z_{i}-\rho_{c} \bar{V}_{i}\right) \vec{\nabla} \psi+T \vec{\nabla} \bar{S}_{i}-\chi \vec{\nabla} \bar{V}_{i}\right] \\
& -\left(\vec{J}_{v}-\sum_{j=1}^{N_{f}} \bar{V}_{j} \vec{J}_{j}^{0}\right) \cdot \sum_{i=0}^{N_{f}} C_{i}\left(T \vec{\nabla} \bar{S}_{i}-\chi \vec{\nabla} \bar{V}_{i}\right)
\end{aligned}
$$


The existence of an electrical potential gradient, $\vec{\nabla} \psi$, can induce motion of ionic solutes. The density of flow of electric charge, $\vec{I}$, due to this motion is defined by

$$
\vec{I}=\sum_{i=1}^{N_{f}} Z_{i} \overrightarrow{J_{i}}
$$

In some applications, it is desirable to separate the electrical potential gradient and the ionic current from other terms in (44). Using (22), (30), (43) and (45), (44) may be written

$$
\begin{aligned}
Q= & -\vec{J}_{v} \cdot\left(\vec{\nabla} P+\rho_{f} g \vec{\nabla} z\right)-\vec{I} \cdot \vec{\nabla} \psi-\sum_{i=1}^{N_{f}} \vec{J}_{i}^{0} \cdot\left[\vec{\nabla} \mu_{i}^{e}+\left(1-\rho_{f} \bar{\nabla}_{i}\right) g \vec{\nabla} z+T \vec{\nabla} \bar{S}_{i}-\chi \vec{\nabla} \bar{V}_{i}\right] \\
& -\left(\vec{J}_{v}-\sum_{j=1}^{N_{f}} \bar{V}_{j} \vec{J}_{j}^{0}\right) \cdot \sum_{i=0}^{N_{f}} C_{i}\left(T \vec{\nabla} \bar{S}_{i}-\chi \vec{\nabla} \bar{V}_{i}\right)
\end{aligned}
$$

If (33) is substituted into (45) and (43) is used, the ionic current, $\vec{I}$, may be expressed in terms of the volume flux, $\vec{J}_{v}$, the diffusional fluxes of solutes, $\vec{J}_{i}^{0}, i=1, \ldots, N_{f}$, and the density of electric charge, $\rho_{c}$ :

$$
\vec{I}=\rho_{c} \vec{J}_{v}+\sum_{i=1}^{N_{f}} \vec{J}_{i}\left(Z_{i}-\rho_{c} \vec{V}_{i}\right)
$$

If now the condition of electrical neutrality $\left(\rho_{c}=0\right)$ is imposed, the ionic current becomes independent of the frame of reference, as pointed out by KATCHALSKY AND CURRAN (1967, p. 141):

$$
\left.\vec{I}\right|_{\rho_{0}=0}=\sum_{i=1}^{N_{l}} Z_{i} \vec{J}_{i}^{0}
$$

With the condition of electrical neutrality, all terms involving $\rho_{c}$ vanish from (44). If the constraint that $\vec{I}=0$ is imposed, (47) shows that all remaining terms involving $\vec{\nabla} \psi$ vanish from (44) and the term $-\vec{I} \cdot \vec{\nabla} \psi$ vanishes from (46). As seen from (48), the conditions $\rho_{c}=0$ and $\vec{I}=0$ introduce a linear, homogeneous dependency into the set of diffusional fluxes $\vec{J}_{i}^{0}, i=1, \ldots, N_{f}$; this point is discussed further in the section on the phenomenological equations.

\section{Summary of this Section}

In summary, the complete set of governing equations is: (4) for mass, (37) for temperature, and (38) for pressure. Terms involving potential energy in the the latter two equations can be expressed by (44) or (46) if the specific potential energy, $\varphi_{i}$, of solute $i$ is given by (39). Further modifications result if the constraints $\rho_{c}=0$ and $\vec{I}=0$ are imposed. These equations extend the governing equations given by FITTs $\left(1962\right.$, p. 53) to open systems and express the mass fluxes in forms $\left(\vec{J}_{v}, \vec{J}_{i}^{\circ}, i=1, \ldots, N_{f}\right)$ most useful in applications to multicomponent, subsurface fluids. The present governing equations are based on the concept of entropy balance, a key concept in TTIP. A parallel development, based on methods of continuum mechanics, has been given by HASSANIZADEH (1986a). 


\section{THE DISSIPATION FUNCTION}

The governing equations, derived in the previous section, relate rates of change of intensive state variables (mass concentrations, temperature, pressure) to fluxes of mass and energy in the system under study. To proceed further, it is necessary to formulate the phenomenological equations that provide mathematical relationships between the fluxes and the forces. As explained in the preceding discussion of TTIP, explicit formulation of the dissipation function is prerequisite to correct identification of the fluxes and forces. This procedure will ensure that the associated phenomenological coefficients satisfy the Onsager reciprocal relations (2). In this section, the dissipation function is formulated for the system under study. In the succeeding section, the fluxes and forces are identified explicitly, and the phenomenological equations are formulated.

\section{General Formulation}

The balance equation for entropy may be written, in general,

$$
\frac{\partial C_{s}}{\partial t}=-\vec{\nabla} \cdot \vec{J}_{s}+\sigma
$$

where $\vec{J}_{8}$ is the flux of entropy through a volume element and $\sigma$ is the local source term of entropy arising from irreversible processes within the volume element; $\sigma$ must be nonnegative. The equivalent balance equation for the system under study may be formed by substituting (4), (18), and (19) into (17). The result after dividing both sides by $T$ is

$$
\begin{aligned}
\frac{\partial C_{i}}{\partial t}= & -\frac{1}{T} \vec{\nabla} \cdot\left[\vec{J}_{q}+\sum_{i=0}^{N_{f}}\left(H_{i}+\varphi_{i}\right) \vec{J}_{i}\right]-\frac{1}{T} \sum_{i=0}^{N_{f}}\left(\mu_{i}+\varphi_{i}\right)\left(-\vec{\nabla} \cdot \vec{J}_{i}+\sum_{f=1}^{R_{f}} M_{i} \nu_{i f} J_{f}+\sum_{r=1}^{R_{r}} M_{i} \nu_{i r} J_{r}\right) \\
& -\frac{1}{T} \sum_{j=1}^{N_{r}}\left(\mu_{j}+\varphi_{j}\right)\left(\sum_{r=1}^{R_{r}} M_{j} \nu_{j r} J_{r}\right) .
\end{aligned}
$$

The affinity, $\tilde{A}_{f}$, of homogeneous reaction, $f$, and the affinity, $\tilde{A}_{r}$, of heterogeneous reaction, $r$, are defined by

$$
\begin{aligned}
& \tilde{A}_{f}=-\sum_{i=0}^{N_{f}} M_{i} \nu_{i f}\left(\mu_{i}+\varphi_{i}\right), \quad f=1, \ldots, R_{f} \\
& \tilde{A}_{r}=-\sum_{i=0}^{N_{f}} M_{i} \nu_{i r}\left(\mu_{i}+\varphi_{i}\right)-\sum_{j=1}^{N_{r}} M_{j} \nu_{j r}\left(\mu_{j}+\varphi_{j}\right), \quad r=1, \ldots, R_{r} .
\end{aligned}
$$

Using (51) and the identity,

$$
\left(\mu_{i}+\varphi_{i}\right) \vec{\nabla} \cdot \vec{J}_{i} \equiv \vec{\nabla} \cdot\left[\left(\mu_{i}+\varphi_{i}\right) \overrightarrow{J_{i}}\right]-\overrightarrow{J_{i}} \cdot \vec{\nabla}\left(\mu_{i}+\varphi_{i}\right)
$$

(50) becomes

$$
\frac{\partial C_{i}}{\partial t}=-\frac{1}{T} \vec{\nabla} \cdot\left[\vec{J}_{q}+\sum_{i=0}^{N_{f}}\left(\bar{H}_{i}-\mu_{i}\right) \vec{J}_{i}\right]-\frac{1}{T} \sum_{i=0}^{N_{f}} \vec{J}_{i} \cdot \vec{\nabla}\left(\mu_{i}+\varphi_{i}\right)+\frac{1}{T} \sum_{f=1}^{R_{f}} J_{f} \tilde{A}_{f}+\frac{1}{T} \sum_{r=1}^{R_{r}} J_{r} \tilde{A}_{r}
$$


Then, using (C-7a) and the identity,

$$
\frac{1}{T} \vec{\nabla} \cdot\left[T\left(\frac{\vec{J}_{q}}{T}+\sum_{i=0}^{N_{f}} \bar{S}_{i} \vec{J}_{i}\right)\right] \equiv \vec{\nabla} \cdot\left(\frac{\vec{J}_{q}}{T}+\sum_{i=0}^{N_{f}} \bar{S}_{i} \vec{J}_{i}\right)+\left(\frac{\vec{J}_{q}}{T}+\sum_{i=0}^{N_{f}} \bar{S}_{i} \vec{J}_{i}\right) \cdot \frac{\vec{\nabla} T}{T}
$$

(53) becomes

$$
\begin{aligned}
\frac{\partial C_{s}}{\partial t}=-\vec{\nabla} \cdot\left(\frac{\vec{J}_{q}}{T}+\sum_{i=0}^{N_{f}} \bar{S}_{i} \vec{J}_{i}\right)-\left(\frac{\vec{J}_{q}}{T}+\sum_{i=0}^{N_{f}} \bar{S}_{i} \vec{J}_{i}\right) \cdot \frac{\vec{\nabla} T}{T}-\frac{1}{T} \sum_{i=0}^{N_{f}} \vec{J}_{i} \cdot \vec{\nabla}\left(\mu_{i}+\varphi_{i}\right) \\
+\frac{1}{T} \sum_{f=1}^{R_{f}} J_{f} \tilde{A}_{f}+\frac{1}{T} \sum_{r=1}^{R_{r}} J_{r} \tilde{A}_{r}
\end{aligned}
$$

Comparing (55) to (49) provides the following identifications:

$$
\vec{J}_{s}=\frac{\vec{J}_{q}}{T}+\sum_{i=0}^{N_{f}} \vec{S}_{i} \vec{J}_{i}
$$

and

$$
T \sigma=\Phi=-\vec{J}_{0} \cdot \vec{\nabla} T-\sum_{i=0}^{N_{f}} \vec{J}_{i} \cdot \vec{\nabla}\left(\mu_{i}+\varphi_{i}\right)+\sum_{f=1}^{R_{f}} J_{f} A_{f}+\sum_{r=1}^{R_{r}} J_{r} A_{r}
$$

where $\Phi$, the dissipation function, represents the local rate of dissipation of Gibbs free energy (the energy available to do work) by irreversible processes (KatchalskY aNd CURRAN, 1967, p. 80). In (56), the entropy flux, $\vec{J}_{8}$, is seen to be composed of a part arising from conductive heat flow and a part representing the advective flux of entropy carried by the mass fluxes. In (57), the dissipation function, $\Phi$, is seen to be the sum of products of fluxes and forces [cf. (1)], and the flux conjugated to the force, $-\vec{\nabla} T$, is the entropy flux, $\vec{J}_{s}$.

Several modifications to (57) are needed to bring the dissipation function into its most useful form. First, the temperature dependence of the chemical potentials, $\mu_{i}, i=0, \ldots, N_{f}$, will be separated from them and incorporated within the term in $\Phi$ involving $-\vec{\nabla} T$. This operation will have the result that the flux in this term will become the heat flux, $\vec{J}_{q}$, and the force will become $-(\vec{\nabla} T) / T$. Second, the mass fluxes, $\vec{J}_{i}, i=0, \ldots, N_{f}$, will be replaced by the volume flux, $\vec{J}_{v}$, and the diffusional fiuxes of solutes, $\vec{J}_{i}^{\circ}$, $i=1, \ldots, N_{f}$ [cf. (22), (30)]. Finally, the particular forms assumed by $\Phi$ in fields of gravitational and electrical potentials will be derived.

The gradients of the chemical potentials of the solution species, $\vec{\nabla} \mu_{i}, i=0, \ldots, N_{f}$, are expanded into components consisting of temperature, pressure, and compositional gradients by substituting (26) and (56) into (57). The result is

$$
\Phi=-\vec{J}_{q} \cdot \frac{\vec{\nabla} T}{T}-\sum_{i=0}^{N_{f}} \vec{J}_{i} \cdot\left(\vec{V}_{i} \vec{\nabla} P+\vec{\nabla} \mu_{i}^{e}+\vec{\nabla} \varphi_{i}\right)+\sum_{f=1}^{R_{f}} J_{f} \tilde{A}_{f}+\sum_{r=1}^{R_{r}} J_{r} \tilde{A}_{r}
$$

The mass fluxes in the laboratory reference frame, $\vec{J}_{i}, i=0, \ldots, N_{f}$, are converted to the volume flux, $\vec{J}_{v}$, and the diffusional solute fluxes, $\vec{J}_{i}^{\circ}, i=1, \ldots, N_{f}$, by substituting (28), (32), and (33) into (58) 
and using (31). The result is

$$
\begin{aligned}
\Phi= & -\vec{J}_{q} \cdot \frac{\vec{\nabla} T}{T}-\vec{J}_{v} \cdot\left(\vec{\nabla} P+\sum_{i=0}^{N_{f}} C_{i} \vec{\nabla} \varphi_{i}\right)-\sum_{i=1}^{N_{f}} \vec{J}_{i}^{0} \cdot\left(\vec{\nabla} \mu_{i}^{e}+\vec{\nabla} \varphi_{i}-\nabla_{i} \sum_{j=0}^{N_{f}} C_{j} \vec{\nabla} \varphi_{j}\right) \\
& +\sum_{f=1}^{R_{f}} J_{f} \tilde{A}_{f}+\sum_{r=1}^{R_{r}} J_{r} \tilde{A}_{r} .
\end{aligned}
$$

To proceed further, the forms of the potential energies, $\varphi_{i}, i=0, \ldots, N_{f}$, and $\varphi_{j}, j=1, \ldots, N_{r}$, must be specified. In fields of gravitational and electrical potentials, the specific potential energies of the solution species are given by (39) and those of the electrically neutral, reactive solids are given simply by

$$
\varphi_{j}=g z, \quad j=1, \ldots, N_{r}
$$

When (39) is substituted into (59), and (42) and (43) are used, the result is

$$
\begin{aligned}
\Phi= & -\vec{J}_{q} \cdot \frac{\vec{\nabla} T}{T}-\vec{J}_{v} \cdot\left(\vec{\nabla} P+\rho_{f} g \vec{\nabla} z+\rho_{c} \vec{\nabla} \psi\right)-\sum_{i=1}^{N_{f}} \vec{J}_{i}^{0} \cdot\left[\vec{\nabla} \mu_{i}^{c}+\left(1-\rho_{f} \nabla_{i}\right) g \vec{\nabla} z+\left(Z_{i}-\rho_{c} \nabla_{i}\right) \vec{\nabla} \psi\right] \\
& +\sum_{f=1}^{R_{f}} J_{f} \tilde{A}_{f}+\sum_{r=1}^{R_{r}} J_{r} \tilde{A}_{r} .
\end{aligned}
$$

As in the case of the governing equations, if the condition of local electrical neutrality $\left(\rho_{c}=0\right)$ is imposed, then all terms involving $\rho_{c}$ vanish from (61) and the dissipation function becomes

$$
\begin{aligned}
\Phi= & -\vec{J}_{q} \cdot \frac{\vec{\nabla} T}{T}-\vec{J}_{v} \cdot\left(\vec{\nabla} P+\rho_{f} g \vec{\nabla} z\right)-\sum_{i=1}^{N_{f}} \vec{J}_{i}^{\circ} \cdot\left[\ddot{\nabla} \mu_{i}^{c}+\left(1-\rho_{f} \nabla_{i}\right) g \vec{\nabla} z+Z_{i} \vec{\nabla} \psi\right] \\
& +\sum_{f=1}^{R_{f}} J_{f} \tilde{A}_{f}+\sum_{r=1}^{R_{r}} J_{r} \tilde{A}_{r} .
\end{aligned}
$$

Equation (62) is the general formulation of the dissipation function for the system with potential energies given by (39) and (60). Next, we consider specific applications of (62).

\section{Nonionic Solutes}

If the solutes are nonionic (i.e., $Z_{i}=0, i=1, \ldots, N_{f}$ ), electrical effects do not contribute to the production of entropy, and the dissipation function (62) becomes

$$
\Phi=-\vec{J}_{q} \cdot \frac{\vec{\nabla} T}{T}-\vec{J}_{v} \cdot\left(\vec{\nabla} P+\rho_{f} g \vec{\nabla} z\right)-\sum_{i=1}^{N_{f}} \vec{J}_{i}^{0} \cdot\left[\vec{\nabla} \mu_{i}^{e}+\left(1-\rho_{f} \bar{V}_{i}\right) g \vec{\nabla} z\right]+\sum_{f=1}^{R_{f}} J_{f} \tilde{A}_{f}+\sum_{r=1}^{R_{r}} J_{r} \tilde{A}_{r}
$$




\section{Ionic Solutes with Electroneutrality and Zero Ionic Current}

Two methods are available for forming the dissipation function for systems containing ionic solutes when $\rho_{c}=0$ and $\vec{I}=0$. The latter condition reduces the number of independent diffusional fiuxes by one.

If the solute cations and anions can be combined unambiguously into one or more neutral compounds, then the system can be described by the dissipation function for a system of nonionic solutes, as in (63). [In this case, $N_{f}$ in (63) would be the number of neutral dissolved compounds.] MiLler $(1959,1966)$ and FitTs $(1962, \$ 7-2)$ have shown the equivalence of the two systems in this case. The description of the nonionic system would involve at least one less diffusional flux than the number of ionic species. For examples, a solution containing $\mathrm{Na}^{+}, \mathrm{K}^{+}$, and $\mathrm{Cl}^{-}$ions would have two nonionic fluxes, those of $\mathrm{NaCl}$ and $\mathrm{KCl}$; a solution obtained by dissolving the compounds $\mathrm{NaNO}_{3}$ and $\mathrm{KCl}$ could have four ionic fluxes, those of $\mathrm{Na}^{+}, \mathrm{K}^{+}, \mathrm{Cl}^{-}$and $\mathrm{NO}_{3}^{-}$, of which three would be independent, or it could have the two fluxes of the original compounds.

On the other hand, combining cations and anions into neutral compounds may be ambiguous or inconvenient, and describing the system in terms of ionic fluxes may be required. In this case, the phenomenological equations are formed from the dissipation function given by (62). These phenomenological equations can be written in several different forms; this point is discussed in the next section.

\section{System with Electrode Processes}

We consider a system containing electrodes inserted in ionic solutions of different concentrations separated by a membrane or a liquid junction. The electrodes are reversible with respect to one of the ions in solution. Ionic diffusional flows can contribute to an ionic current, $\vec{I}$, given by (45), driven by an electromotive force, $-\vec{\nabla} \psi$, between the electrodes. In this case, the appearance of certain electrokinetic phenomena (discussed in the next section) makes desirable the separation of $\vec{\nabla} \psi$ and $\vec{I}$ from other terms in (62). To do this, (47) can be substituted into (61) or (48) into (62); in either case, the result is

$$
\begin{aligned}
\Phi= & -\vec{J}_{q} \cdot \frac{\vec{\nabla} T}{T}-\vec{J}_{v} \cdot\left(\vec{\nabla} P+\rho_{f} g \vec{\nabla} z\right)-\sum_{i=1}^{N_{f}} \vec{J}_{i}^{0} \cdot\left[\vec{\nabla} \mu_{i}^{e}+\left(1-\rho_{f} \bar{V}_{i}\right) g \vec{\nabla} z\right]-\vec{I} \cdot \vec{\nabla} \psi \\
& +\sum_{f=1}^{R_{f}} J_{f} \tilde{A}_{f}+\sum_{r=1}^{R_{r}} J_{r} \tilde{A}_{r} .
\end{aligned}
$$

The new term, $-\vec{I} \cdot \vec{\nabla} \psi$, represents the dissipation of the system's free energy (conversion of free energy to entropy) by electrical conduction.

Equations (62), (63), and (64) are the forms of the dissipation function, $\Phi$, that will be used subsequently in this work.

\section{The Chemical Affinities}

It remains to specify the forms of the chemical affinities under the assumption of gravitational and electrical potential energies. Substituting (39) and (60) into (51) gives

$$
A_{f}=-\sum_{i=0}^{N_{f}} M_{i} \nu_{i f} \mu_{i}-g z \sum_{i=0}^{N_{f}} M_{i} \nu_{i f}-\psi \sum_{i=0}^{N_{f}} M_{i} \nu_{i f} Z_{i}, f=1, \ldots, R_{f}
$$

and

$$
\tilde{A}_{r}=-\sum_{i=0}^{N_{g}} M_{i} \nu_{i r} \mu_{i}-\sum_{j=1}^{N_{r}} M_{j} \nu_{j r} \mu_{j}-g z\left(\sum_{i=0}^{N_{g}} M_{i} \nu_{i r}+\sum_{j=1}^{N_{r}} M_{j} \nu_{j r}\right)
$$




$$
-\psi \sum_{i=0}^{N_{f}} M_{i} \nu_{i r} Z_{i}, \quad r=1, \ldots, R_{r}
$$

However, conservation of mass in chemical reactions requires that

$$
\begin{array}{r}
\sum_{i=0}^{N_{j}} M_{i} \nu_{i j}=0, \quad f=1, \ldots, R_{f}, \\
\sum_{i=0}^{N_{j}} M_{i} \nu_{i r}+\sum_{j=1}^{N_{r}} M_{j} \nu_{j r}=0, \quad r=1, \ldots, R_{r},
\end{array}
$$

and conservation of electrical charge requires that

$$
\begin{aligned}
& \sum_{i=0}^{N_{f}} M_{i} \nu_{i f} Z_{i}=0, \quad f=1, \ldots, R_{f}, \\
& \sum_{i=0}^{N_{f}} M_{i} \nu_{i r} Z_{i}=0, \quad r=1, \ldots, R_{r}
\end{aligned}
$$

Then, using (66) and (67) in (65) gives

$$
\tilde{A}_{f}=-\sum_{i=0}^{N_{f}} M_{i} \nu_{i f} \mu_{i}, \quad f=1, \ldots, R_{f}
$$

and

$$
\tilde{A}_{r}=-\sum_{i=0}^{N_{f}} M_{i} \nu_{i r} \mu_{i}-\sum_{j=1}^{N_{r}} M_{j} \nu_{j r} \mu_{j}, \quad r=1, \ldots, R_{r}
$$

\section{THE PHENOMENOLOGICAL EQUATIONS}

With the dissipation function for the system under consideration at hand in the form given in general by (1), the fluxes and forces can be identified and used to formulate the phenomenological equations according to the second postulate of TTIP as stated in (2). Then, by the third postulate, the phenomenological coefficients are assumed to satisfy the reciprocal relations stated generally in (3).

We adopt Curie's theorem and the extension thereof to systems with symmetries lower than isotropic, i.e., orthorhombic, tetragonal, and cubic (CARNAHAN, 1976). This allows the scalar chemical reaction rates to be uncoupled from the vector fluxes of heat, mass, and electric charge; the result is a considerable simplification of the phenomenological equations.

We follow the sequence of specific applications used in the preceding section. For clarification, Table 1 provides names of all direct and coupled vector processes considered here, arranged according to driving forces and resulting fluxes. 
TABLE 1. Direct and Coupled Vector Processes

\begin{tabular}{|c|c|c|c|c|c|}
\hline \multirow{2}{*}{ Fluxes } & \multicolumn{5}{|c|}{ Driving Forces } \\
\hline & $-(\vec{\nabla} T) / T$ & $\overrightarrow{X_{p}^{*}}$ & $\bar{X}_{c, 1} *$ & $\ldots \vec{X}_{c, N_{1}}{ }^{*}$ & $-\vec{\nabla} \psi$ \\
\hline Heat & Fourier's law & thermal filtration & $\begin{array}{r}\text { diffusior } \\
\text { (Du }\end{array}$ & $\begin{array}{l}\text { thermal effect } \\
\text { our effect) }\end{array}$ & Peltier effect \\
\hline $\begin{array}{l}\text { Volume } \\
\text { Mass of solute } 1\end{array}$ & $\begin{array}{l}\text { thermal osmosis } \\
\text { thermal diffusion } \\
\text { (Sorêt effect) }\end{array}$ & $\begin{array}{c}\text { Darcy's law } \\
\text { ultrafiltration }\end{array}$ & $\begin{array}{l}\text { chem } \\
\text { Fick's } \\
\text { law }\end{array}$ & $\begin{array}{l}\text { cal osmosis } \\
\ldots \text { coupled } \\
\text { diffusion }\end{array}$ & $\begin{array}{l}\text { electro-osmosis } \\
\text { electrophoresis }\end{array}$ \\
\hline Mass of solute $N_{f}$ & $\begin{array}{l}\text { thermal diffusion } \\
\text { (Sorêt effect) }\end{array}$ & ultrafiltration & $\begin{array}{l}\text { coupled } \\
\text { diffusion }\end{array}$ & $\begin{array}{cc}\ldots & \ldots \\
\ldots & \text { Fick's } \\
\text { law }\end{array}$ & electrophoresis \\
\hline Electric charge & Seebeck effect & streaming current & sedimer & ation current & Ohm's law \\
\hline
\end{tabular}

${ }^{*}$ Defined in equation (70).

\section{Nonionic Solutes}

Using the dissipation function given in (63), the phenomenological equations for the vector transport processes are

$$
\begin{aligned}
& \vec{J}_{q}=-\mathrm{L}_{q q} \cdot \frac{\vec{\nabla} T}{T}+\mathrm{L}_{q v} \cdot \vec{X}_{p}+\sum_{i=1}^{N_{f}} \mathrm{~L}_{q i} \cdot \vec{X}_{c, i}, \\
& \vec{J}_{v}=-\mathrm{L}_{v q} \cdot \frac{\vec{\nabla} T}{T}+\mathrm{L}_{v v} \cdot \vec{X}_{p}+\sum_{i=1}^{N_{f}} \mathrm{~L}_{v i} \cdot \vec{X}_{c, i}, \\
& \vec{J}_{i}^{\circ}=-\mathrm{L}_{i q} \cdot \frac{\vec{\nabla} T}{T}+\mathrm{L}_{i v} \cdot \vec{X}_{p}+\sum_{j=1}^{N_{f}} \mathrm{~L}_{i j} \cdot \vec{X}_{c, j}, \quad i=1, \ldots, N_{f},
\end{aligned}
$$

where $N_{f}$ is the number of nonionic (uncharged) solutes and and the forces are defined by

$$
\begin{aligned}
\vec{X}_{p} & =-\left(\vec{\nabla} P+\rho_{\rho} g \vec{\nabla} z\right), \\
\vec{X}_{c, j} & =-\left[\vec{\nabla} \mu_{j}^{c}+\left(1-\rho_{j} \bar{V}_{j}\right) g \vec{\nabla} z\right] .
\end{aligned}
$$

We note that in (69a) the term containing $L_{\varphi q}$ is an analog of Fourier's law, in (69b) the term containing $L_{v v}$ is an analog of Darcy's law, and in $(69 \mathrm{c})$ the terms containing $L_{i l}$ are analogs of Fick's law. The reciprocal relations for the vector processes are

$$
\begin{aligned}
& L_{q u}=L_{v q} \\
& L_{q i}=L_{i q}, \quad L_{v i}=L_{i v}, \quad i=1, \ldots, N_{f}, \\
& L_{i j}=L_{j i}, \quad i, j=1, \ldots, N_{f}, \quad i \neq j .
\end{aligned}
$$

We note that the phenomenological coefficients for the vector processes are, in general, symmetric tensors of rank two (DE Groot and Mazur, 1962, Chap. 6, \$2; Carnahan, 1976). 
The phenomenological equations for the scalar chemical reaction rates are

$$
\begin{gathered}
J_{f}=\sum_{k=1}^{R_{f}} L_{f k} \tilde{A}_{k}, \quad f=1, \ldots, R_{f}, \\
J_{r}=\sum_{l=1}^{R_{r}} L_{r l} \tilde{A}_{l}, \quad r=1, \ldots, R_{r}
\end{gathered}
$$

where

$$
\begin{aligned}
& \tilde{A}_{k}=-\sum_{i=0}^{N_{f}} M_{i} \nu_{i k} \mu_{i}, \quad k=1, \ldots, R_{f}, \\
& \tilde{A}_{l}=-\sum_{i=0}^{N_{f}} M_{i} \nu_{i l} \mu_{i}-\sum_{j=1}^{N_{r}} M_{j} \nu_{j l} \mu_{j}, \quad l=1, \ldots, R_{r}
\end{aligned}
$$

The reciprocal relations for the reaction rates are

$$
\begin{aligned}
L_{f k} & =L_{k f}, \quad f, k=1, \ldots, R_{f}, \quad f \neq k, \\
L_{r l} & =L_{l r}, \quad r, l=1, \ldots, R_{r}, \quad r \neq l .
\end{aligned}
$$

The phenomenological coefficients appearing in (72) and (74) are scalars.

The phenomenological coefficients $L_{q q}, L_{v v}$, and $L_{i j}, i, j=1, \ldots, N_{f}$, can be related to the practical transport coefficients $K$ (thermal conductivity), $k$ (permeability), and $D_{i j}$ (diffusion/dispersion coefficient) appearing in Fourier's law, Darcy's law, and Fick's law, the last generalized to include cross diffusion:

$$
\begin{aligned}
& \vec{J}_{q}=-K \cdot \vec{\nabla} T \quad \text { (Fourier), } \\
& \vec{J}_{v}=-\frac{\rho_{j} g}{\mu_{f}} k \cdot \vec{\nabla} h \quad \text { (Darcy), } \\
& \vec{J}_{i}^{0}=-\sum_{j=1}^{N_{f}} \mathrm{D}_{i j} \cdot \vec{\nabla} C_{j}, \quad i=1, \ldots, N_{f} \quad \text { (Fick), }
\end{aligned}
$$

where $\mu_{f}$ is the dynamic viscosity of the fluid phase and $h$ is the hydraulic head, defined by (FREEzE AND CHERRY, 1979, Chap. 2):

$$
h=\frac{P}{\rho_{f} g}+z .
$$

Comparing (75a) to (69a) provides the relationship

$$
\mathrm{L}_{q q}=\mathrm{K} T \text {. }
$$

Comparing (75b) and (76) to (69b) and (70a) shows that the forces $-\rho \rho g \vec{\nabla} h$ and $-\vec{X}_{p}$ are not equal unless the factor $\rho_{f} g$ is held constant; with this constraint, the coefficients for volume flow are related by

$$
L_{v v}=\mu_{f}^{-1} k \text {. }
$$

To relate the phenomenological coefficients $L_{i j}$ to the diffusion/dispersion coefficients $D_{i j}$, the forces $-\vec{X}_{c, j}$ and $-\vec{\nabla} C_{j}$ must be made equivalent. The force $-\vec{X}_{c, j}$ contains a term, $\left(1-\rho_{j} \vec{\nabla}_{j}\right) g \vec{\nabla} z$, that is 
not accounted for by Fick's law. Groenevelt aND BoLt (1969) attribute this term to the difference in gravitational forces acting on a given mass of solute and the mass of fluid displaced by the solute. This term is usually very small relative to the diffusional force, $-\vec{\nabla} \mu_{j}^{e}$. [GroENEVELT AND BoLT (1969) provide a numerical example comparing the two forces.] The remaining thermodynamic force, $-\vec{\nabla} \mu_{j}^{e}$, can be related to the practical force, $-\vec{\nabla} C_{j}$, by:

$$
\vec{\nabla} \mu_{j}^{e}=\sum_{k=1}^{N_{f}} \mu_{j k} \vec{\nabla} C_{k}, j=1, \ldots, N_{f}
$$

where

$$
\mu_{j k}=\frac{\partial \mu_{j}^{e}}{\partial C_{k}}, \quad j, k=1, \ldots, N_{l}
$$

For the special case of an ideal solution (Katchalsky and CURRAN, 1967, $\$ 5.4$ ),

$$
\begin{aligned}
& \mu_{j j}=\frac{R T}{M_{j} C_{j}} \\
& \mu_{j k}=0, \quad j \neq k .
\end{aligned}
$$

Then, equating $(75 c)$ to the part of $(69 c)$ associated with the force $-\vec{X}_{c, j}$, neglecting the buoyancy term in $\vec{X}_{c, j}$, and using (79) give

$$
\vec{J}_{i}^{0}=\sum_{j=1}^{N_{j}} \mathrm{~L}_{i j} \cdot \sum_{k=1}^{N_{j}} \mu_{j k} \vec{\nabla} C_{k}=\sum_{j=1}^{N_{l}} \mathrm{D}_{i j} \cdot \vec{\nabla} C_{j}
$$

Equating multipliers of the same $\vec{\nabla} C_{j}$ gives

$$
\mathrm{D}_{i j}=\sum_{k=1}^{N_{f}} \mathrm{~L}_{i k} \mu_{k j}
$$

or, in matrix notation,

$$
D=L \cdot \mu
$$

from which is obtained

$$
L=D \cdot \mu^{-1}
$$

If all solutes form ideal solutions, (85) becomes

$$
L_{i j}=\frac{M_{j} C_{j}}{R T} \mathrm{D}_{i j}, \quad i, j=1, \ldots, N_{\rho}
$$

Experiments have confirmed the reciprocal relations for diffusion in fluids and have shown that the phenomenological coefficients for coupled processes, $L_{i j}, i \neq j$, can be significant relative to the coefficients for direct processes, $L_{i i}$ (e.g., MiLlER, 1965). 
HASSANIZADEH (1986b) and HASSANizadeh AND LeIJNSE (1988) used the methods of continuum mechanics to derive equations similar to $(69 \mathrm{~b})$ and $(69 \mathrm{c})$, but lacking the coupling to the gradient of temperature.

\section{Ionic Solutes with Electroneutrality and Zero Ionic Current}

If all solute ions have been combined into one or more neutral compounds, then the phenomenological equations and reciprocal relations are those given by (69)-(74).

If the system is described in terms of ionic fluxes, alternative representations of the phenomenological equations describing the vector processes are available. Which of these representations is most useful in a given situation will depend on the types of measurements used to determine values of the phenomenological coefficients and, more specifically, on the role that the electrical potential gradient, $\vec{\nabla} \psi$, is intended to play in the analysis of a system's behavior. In the following, we begin with the most fundamental representation in terms of a set of independent fluxes. We then discuss another representation based on a set of dependent fiuxes using $-\vec{\nabla} \psi$ explicitly as a driving force. We show how the phenomenological coefficients of the two representations are related, and we show that the reciprocal relations are valid in the case involving dependent fluxes.

From (48) it is seen that the condition of electrical neutrality $\left(\rho_{c}=0\right)$ and the condition of zero ionic current,

$$
\sum_{i=1}^{N_{f}} Z_{i} \vec{J}_{i}^{\circ}=0
$$

impose a homogeneous linear dependency on the set of diffusional fluxes, $\vec{J}_{i}^{\circ}, i=1, \ldots, N_{f}$. In order to guarantee validity of the reciprocal relations (assuming validity of the three fundamental postulates of TTIP), the phenomenological equations for our system must be posed in terms of a set of independent fluxes [cf. discussion following (3)]. The required set is found by using (87) to eliminate one flux; we choose here to eliminate the $N_{f}$-th flux, thus:

$$
\vec{J}_{N_{j}}^{0}=-\sum_{i=1}^{N_{f}-1} \frac{Z_{i}}{Z_{N_{f}}} \vec{J}_{i}^{\circ}
$$

with the stipulation that $Z_{N j} \neq 0$. Equation (88) eliminates one phenomenological equation, resulting in a number $\left(N_{f}-1\right)$ of phenomenological equations equal to the number of independent diffusional fluxes. This elimination is a reflection of the important physical constraint that, in order to maintain local electrical neutrality, the motion of one ionic species must be determined solely by the independent motions of all other ionic species. When (88) is substituted for $\vec{J}_{N_{f}}^{\circ}$ in (62), the dissipation function becomes

$$
\Phi=-\vec{J}_{q} \cdot \frac{\vec{\nabla} T}{T}+\vec{J}_{v} \cdot \vec{X}_{p}+\sum_{i=1}^{N_{f}-1} \vec{J}_{i}^{0} \cdot\left(\vec{X}_{c, i}-\frac{Z_{i}}{Z_{N_{f}}} \vec{X}_{c, N_{f}}\right)+\sum_{f=1}^{R_{f}} J_{f} \tilde{A}_{f}+\sum_{r=1}^{R_{r}} J_{r} \tilde{A}_{r}
$$

Terms involving $\vec{\nabla} \psi$ do not appear in (89). This is consistent with the observation that when $\vec{I}=0$ the contribution to $\Phi$ by the sum of terms involving $\vec{\nabla} \psi$ is identically zero in (62). However, we note that 
the diffusional forces in (89) can be written in an equivalent form that formally includes $\vec{\nabla} \psi$ :

$$
\vec{X}_{c, i}-\frac{Z_{i}}{Z_{N_{f}}} \vec{X}_{c, N_{f}} \equiv\left(\vec{X}_{c, 1}-Z_{i} \vec{\nabla} \psi\right)-\frac{Z_{i}}{Z_{N_{f}}}\left(\vec{X}_{c, N_{s}}-Z_{N_{f}} \vec{\nabla} \psi\right)
$$

The phenomenological equations corresponding to the dissipation function (89) are

$$
\begin{aligned}
& \vec{J}_{q}=-L_{q q} \cdot \frac{\vec{\nabla} T}{T}+L_{q v} \cdot \vec{X}_{p}+\sum_{i=1}^{N_{f}-1} L_{q i} \cdot\left(\vec{X}_{c, i}-\frac{Z_{i}}{Z_{N_{j}}} \vec{X}_{c, N_{j}}\right) \\
& \vec{J}_{v}=-L_{v q} \cdot \frac{\vec{\nabla} T}{T}+L_{v v} \cdot \vec{X}_{p}+\sum_{i=1}^{N_{j}-1} L_{v i} \cdot\left(\vec{X}_{c, i}-\frac{Z_{i}}{Z_{N_{j}}} \vec{X}_{c, N_{j}}\right) \\
& \vec{J}_{i}^{0}=-L_{i q} \cdot \frac{\vec{\nabla} T}{T}+L_{i v} \cdot \vec{X}_{p}+\sum_{j=1}^{N_{j}-1} L_{i j} \cdot\left(\vec{X}_{c, j}-\frac{Z_{j}}{Z_{N_{j}}} \vec{X}_{c, N_{j}}\right), \quad i=1, \ldots, N_{f}-1 .
\end{aligned}
$$

Using (91c) in (88) gives for $\vec{J}_{N_{f}}^{\circ}$

$$
\begin{aligned}
\vec{J}_{N_{j}}^{\circ}=\left(\sum_{i=1}^{N_{f}-1} \frac{Z_{i}}{Z_{N_{f}}} L_{i q}\right) \cdot \frac{\vec{\nabla} T}{T} & -\left(\sum_{i=1}^{N_{f}-1} \frac{Z_{i}}{Z_{N_{j}}} L_{i v}\right) \cdot \vec{X}_{p} \\
& -\sum_{i=1}^{N_{f}-1} \sum_{j=1}^{N_{j}-1} \frac{Z_{i}}{Z_{N_{j}}} L_{i j} \cdot\left(\vec{X}_{c_{j} j}-\frac{Z_{j}}{Z_{N_{f}}} \vec{X}_{c, N_{j}}\right)
\end{aligned}
$$

a result that will be used later. The reciprocal relations associated with the vector processes in (91) are

$$
\begin{aligned}
& \mathrm{L}_{q v}=\mathrm{L}_{v q}, \\
& \mathrm{~L}_{q i}=\mathrm{L}_{i q,}, \quad \mathrm{~L}_{v i}=\mathrm{L}_{i v}, \quad i=1, \ldots, N_{f}-1, \\
& \mathrm{~L}_{i j}=\mathrm{L}_{j i}, \quad i, j=1, \ldots, N_{\rho}-1, \quad i \neq j .
\end{aligned}
$$

The phenomenological equations for the chemical reaction rates in (89) and the associated reciprocal relations are given by (72)-(74). We note that, when (93) is considered, the phenomenological equations for the vector processes, (91), contain $\frac{1}{2}\left(N_{f}+1\right)\left(N_{f}+2\right)$ independent phenomenological coefficients.

We consider next another form in which the phenomenological equations have been expressed in the literature. This form appears to have evolved in response to a perceived need to provide explicit consideration of the electrical potential gradient, $\vec{\nabla} \psi$, in systems in which no electrochemical processes involving transfer of electrons are occurring and no electric field is applied from an external source. The form is based on a set of linearly dependent diffusional fluxes.

The literature contains several discussions about the physical meaning and origin of the electrical potential, $\psi$, in the systems being considered here. In his introduction to an account of computer simulations of liquid junction potentials, HAFEMANN (1965) stated that the assumption of electroneutrality throughout a liquid junction (a system of diffusing ions) "... is self-contradictory because there can be no potential difference if there is no charge separation." HAFEMANN's calculations excluded electroneutrality as a prior condition, and gave the result that an initially sharp liquid junction engendered a transitory charge separation and an electrical potential that rose to a steady value. The rise time of the potential 
was less than $10^{-8} \mathrm{~s}$ in HAFEMANN's simulations, while the time required for the magnitude of the charge imbalance to decline to very small values (less than 0.1 percent excess charge) was of the order of $10^{-7}$ s. HAFEMANN concluded that "... the electroneutrality assumption is approximately valid for the time scales on which experiments are usually performed." Thus, in HAFEMANN's model the potential, $\psi$, arose directly from a macroscopic separation of charge, and electroneutrality was at best an approximation. A somewhat different point of view was advanced by ANDERson AND GrAF (1976), who explained the potential as follows:

"In the absence of an applied electrical field, any migration of ions that destroys electrical neutrality will create an internal electrical field. Reciprocally, this virtual field will act to counter any migration of ions that does not preserve local electrical neutrality."

Thus, in ANDERSON AND GRAF's view, electroneutrality is sacrosanct and is preserved by the electrical potential, $\psi$, which exists in effect but not in actual fact. The idea that the potential acts to preserve electroneutrality (instead of arising directly from a macroscopic imbalance of electric charge) was adopted by LASAGA (1979), who recognized that a charge separation must exist on a microscopic level even though electroneutrality exists on a macroscopic level. In LASAGA's view, variations of charge density occur in the vicinity of ions, and diffusion causes the distribution of ionic charges surrounding each ion to be asymmetric. Then the quantity $-z_{i} \vec{\nabla} \psi$ is the resultant force exerted on an ion having electric charge $z_{i}$ by coulombic interactions with all other ions in solution. This view of $\psi$ corresponds exactly with the model of ionic interaction used in the Debye-Hückel theory of electrolyte solutions (e.g., LEWIS AND RANDALL, 1961, pp. 335-337) and provides a physical basis for $\psi$ while retaining the macroscopic reality of electroneutrality.

When the phenomenological equations are formed directly from the dissipation function given by (62), the following results are obtained for the vector fluxes:

$$
\begin{aligned}
& \vec{J}_{q}=-\mathrm{L}_{q q}^{\prime} \cdot \frac{\vec{\nabla} T}{T}+\mathrm{L}_{q v}^{\prime} \cdot \vec{X}_{p}+\sum_{i=1}^{N_{f}} \mathrm{~L}_{q i}^{\prime} \cdot\left(\vec{X}_{c, i}-Z_{i} \vec{\nabla} \psi\right) \\
& \vec{J}_{v}=-\mathrm{L}_{v q}^{\prime} \cdot \frac{\vec{\nabla} T}{T}+\mathrm{L}_{v v}^{\prime} \cdot \vec{X}_{p}+\sum_{i=1}^{N_{f}} \mathrm{~L}_{v i}^{\prime} \cdot\left(\vec{X}_{c, i}-Z_{i} \vec{\nabla} \psi\right), \\
& \vec{J}_{i}^{0}=-\mathrm{L}_{i q}^{\prime} \cdot \frac{\vec{\nabla} T}{T}+\mathrm{L}_{i v}^{\prime} \cdot \vec{X}_{p}+\sum_{j=1}^{N_{f}} \mathrm{~L}_{i j}^{\prime} \cdot\left(\vec{X}_{c, j}-Z_{j} \vec{\nabla} \psi\right), \quad i=1, \ldots, N_{f}
\end{aligned}
$$

These phenomenological equations include a set of fluxes, $\vec{J}_{i}^{\circ}, i=1, \ldots, N_{f}$, among which exists a homogeneous, linear dependency arising from (87). The equations contain the as yet unknown forces, $-Z_{i} \vec{\nabla} \psi$. In principle, the electrical potential, $\psi$, can be found by solving an associated Poisson's equation, the method used in the Debye-Hückel theory (LEWIS AND RANDALL, 1961, pp. 335-337). In practice (e.g., LASAGA, 1979; Sharland et al., 1987), the unknown forces have been evaluated by use of the condition of zero ionic current. Thus, in the present system (94c) could be substituted into (87) and the result solved for $\vec{\nabla} \psi$, giving

$$
\vec{\nabla} \psi=\left(\sum_{i=1}^{N_{f}} \sum_{j=1}^{N_{f}} Z_{i} Z_{j} \mathrm{~L}_{i j}^{\prime}\right)^{-1} \cdot\left(-\sum_{i=1}^{N_{f}} Z_{i} \mathrm{~L}_{i q}^{\prime} \cdot \frac{\vec{\nabla} T}{T}+\sum_{i=1}^{N_{f}} Z_{i} \mathrm{~L}_{i v}^{\prime} \cdot \vec{X}_{p}+\sum_{i=1}^{N_{f}} \sum_{j=1}^{N_{f}} Z_{i} \mathrm{~L}_{i j}^{\prime} \cdot \vec{X}_{c, j}\right)
$$

The phenomenological equations (94) have same form as (91) and (92) when account is taken of (90). Term by term comparison of the two sets of phenomenological equations provides the following relations 
between their coefficients:

$$
\begin{aligned}
& \mathrm{L}_{q q}^{\prime}=\mathrm{L}_{q q}, \quad \mathrm{~L}_{q v}^{\prime}=\mathrm{L}_{q v}, \quad \mathrm{~L}_{v q}^{\prime}=\mathrm{L}_{v q}, \quad \mathrm{~L}_{v v}^{\prime}=\mathrm{L}_{v v}, \\
& \mathrm{~L}_{q i}^{\prime}=\mathrm{L}_{q i}, \quad \mathrm{~L}_{i q}^{\prime}=\mathrm{L}_{i q}, \quad \mathrm{~L}_{v i}^{\prime}=\mathrm{L}_{v i}, \quad \mathrm{~L}_{i v}^{\prime}=\mathrm{L}_{i v}, \quad i=1, \ldots, N_{f}-1, \\
& \mathrm{~L}_{i j}^{\prime}=\mathrm{L}_{i j}, \quad i, j=1, \ldots, N_{f}-1,
\end{aligned}
$$

and

$$
\begin{aligned}
& \mathrm{L}_{q N_{f}}^{\prime}=-\sum_{i=1}^{N_{f}-1} \frac{Z_{i}}{Z_{N_{j}}} \mathrm{~L}_{q i}, \quad \mathrm{~L}_{N_{f}}^{\prime}=-\sum_{i=1}^{N_{f}-1} \frac{Z_{i}}{Z_{N_{f}}} \mathrm{~L}_{i q}, \\
& \mathrm{~L}_{v N_{j}}^{\prime}=-\sum_{i=1}^{N_{f}-1} \frac{Z_{i}}{Z_{N_{f}}} \mathrm{~L}_{v i}, \quad \mathrm{~L}_{N_{f} v}^{\prime}=-\sum_{i=1}^{N_{f}-1} \frac{Z_{i}}{Z_{N_{f}}} \mathrm{~L}_{i v}, \\
& \mathrm{~L}_{i N_{f}}^{\prime}=-\sum_{j=1}^{N_{f}-1} \frac{Z_{i}}{Z_{N_{f}}} \mathrm{~L}_{i j}, \quad \mathrm{~L}_{N_{f} i}^{\prime}=-\sum_{j=1}^{N_{f}-1} \frac{Z_{i}}{Z_{N_{f}}} \mathrm{~L}_{j i}, \quad i=1, \ldots, N_{f}-1, \\
& \mathrm{~L}_{N_{f} N_{f}}^{\prime}=\sum_{i=1}^{N_{f}-1} \sum_{j=1}^{N_{f}-1} \frac{Z_{i} Z_{j}}{Z_{N_{f}}^{2}} \mathrm{~L}_{i j} .
\end{aligned}
$$

Assuming validity of the fundamental postulates of TTIP and, thus, of the reciprocal relations in (93), (96) and (97) show that the reciprocal relations are valid also for the matrix of phenomenological coefficients, [ $\left.L^{\prime}\right]$, appearing in (94). Indeed, the reciprocal relations have been confirmed experimentally in binary systems described by phenomenological equations of the type of (94c), but at constant temperature and pressure (e.g., MiLLER, 1974; Miller et al., 1984; and references therein). Furthermore, it is seen that, if (96) is used in (97), then the number of independent phenomenological coefficients in (94) is the same as in (91), namely $\frac{1}{2}\left(N_{f}+1\right)\left(N_{f}+2\right)$. DE GROOT AND MAzUR (1962, Chap. 6, \$3) provide general rules on the validity of the reciprocal relations when dependencies exist among the fluxes and forces.

\section{System with Electrode Processes}

In this system the electrical potential, $\psi$, may be imposed by an external source as well as arise from electrochemical processes at electrodes. The electrodes provide means for continuous measurement of either $\psi$ or the ionic current, $\vec{I}$. The dissipation function for this system given by (64) includes $N_{f}$ diffusional fluxes and $\vec{I}$; from (48), there is a linear dependency among this set of fluxes. We shall remove the dependency to ensure validity of the reciprocal relations for the resulting phenomenological equations and then compare these results to the phenomenological equations derived directly from (64) that include the dependency.

We eliminate the dependency from the mass and electrical fluxes by solving (48) for the $N_{f}$-th diffusional flux:

$$
\vec{J}_{N_{f}}^{\circ}=\frac{\vec{I}}{Z_{N_{f}}}-\sum_{i=1}^{N_{f}-1} \frac{Z_{i}}{Z_{N_{f}}} \vec{J}_{i}^{\circ}
$$

Substituting (98) into (64) gives the dissipation function based on a set of independent fluxes, $\vec{J}_{i}^{\circ}$, $i=1, \ldots, N_{f}-1$, and $\vec{I}$ :

$$
\Phi=-\vec{J}_{q} \cdot \frac{\vec{\nabla} T}{T}+\vec{J}_{v} \cdot \vec{X}_{p}+\sum_{i=1}^{N_{f}-1} \vec{J}_{i}^{\circ} \cdot\left(\vec{X}_{e, i}-\frac{Z_{i}}{Z_{N_{f}}} \vec{X}_{c, N_{f}}\right)-\vec{I} \cdot\left(\vec{\nabla} \psi-\frac{\vec{X}_{e, N_{f}}}{Z_{N_{f}}}\right)
$$




$$
+\sum_{f=1}^{R_{f}} J_{f} \tilde{A}_{f}+\sum_{r=1}^{R_{r}} J_{r} \tilde{A}_{r} .
$$

It follows that the independent phenomenological equations for vector processes are:

$$
\begin{array}{r}
\vec{J}_{q}=-\mathrm{L}_{q q} \cdot \frac{\vec{\nabla} T}{T}+\mathrm{L}_{q v} \cdot \vec{X}_{p}+\sum_{i=1}^{N_{f}-1} \mathrm{~L}_{q i} \cdot\left(\vec{X}_{c, i}-\frac{Z_{i}}{Z_{N_{f}}} \vec{X}_{c, N_{f}}\right)-\mathrm{L}_{q e} \cdot\left(\vec{\nabla} \psi-\frac{\vec{X}_{c, N_{f}}}{Z_{N_{f}}}\right), \\
\vec{J}_{v}=-\mathrm{L}_{v q} \cdot \frac{\vec{\nabla} T}{T}+\mathrm{L}_{v v} \cdot \vec{X}_{p}+\sum_{i=1}^{N_{f}-1} \mathrm{~L}_{v i} \cdot\left(\vec{X}_{c, i}-\frac{Z_{i}}{Z_{N_{f}}} \vec{X}_{c, N_{f}}\right)-\mathrm{L}_{v e} \cdot\left(\vec{\nabla} \psi-\frac{\vec{X}_{c, N_{f}}}{Z_{N_{f}}}\right), \\
\vec{J}_{i}^{\circ}=-\mathrm{L}_{i q} \cdot \frac{\vec{\nabla} T}{T}+\mathrm{L}_{i v} \cdot \vec{X}_{p}+\sum_{j=1}^{N_{f}-1} \mathrm{~L}_{i j} \cdot\left(\vec{X}_{c, j}-\frac{Z_{j}}{Z_{N_{f}}} \vec{X}_{c, N_{f}}\right)-\mathrm{L}_{i e} \cdot\left(\vec{\nabla} \psi-\frac{\vec{X}_{c, N_{f}}}{Z_{N_{f}}}\right), \\
\vec{I}=-\mathrm{L}_{e q} \cdot \frac{\vec{\nabla} T}{T}+\mathrm{L}_{e v} \cdot \vec{X}_{p}+\sum_{i=1}^{N_{f}-1} \mathrm{~L}_{e i} \cdot\left(\vec{X}_{c, i}-\frac{Z_{i}}{Z_{N_{f}}} \vec{X}_{c, N_{f}}\right)-\mathrm{L}_{e e} \cdot\left(\vec{\nabla} \psi-\frac{\vec{X}_{c, N_{f}}}{Z_{N_{f}}}\right) .
\end{array}
$$

$\vec{J}_{, N_{f}}^{0}$ is found by substituting (100c) and (100d) into (98); the result is

$$
\begin{aligned}
\vec{J}_{N_{j}}^{0} & =\left(\sum_{i=1}^{N_{f}-1} \frac{Z_{i}}{Z_{N_{f}}} \mathrm{~L}_{i q}-\frac{\mathrm{L}_{e q}}{Z_{N_{j}}}\right) \cdot \frac{\vec{\nabla} T}{T}-\left(\sum_{i=1}^{N_{f}-1} \frac{Z_{i}}{Z_{N_{f}}} \mathrm{~L}_{i v}-\frac{\mathrm{L}_{e v}}{Z_{N_{f}}}\right) \cdot \vec{X}_{p} \\
& -\sum_{i=1}^{N_{f}-1}\left(\sum_{j=1}^{N_{f}-1} \frac{Z_{j}}{Z_{N_{f}}} \mathrm{~L}_{j i}-\frac{\mathrm{L}_{e i}}{Z_{N_{f}}}\right) \cdot\left(\vec{X}_{c, i}-\frac{Z_{i}}{Z_{N_{f}}} \vec{X}_{c, N_{f}}\right)+\left(\sum_{i=1}^{N_{f}-1} \frac{Z_{i}}{Z_{N_{f}}} \mathrm{~L}_{i e}-\frac{\mathrm{L}_{e e}}{Z_{N_{f}}}\right) \cdot\left(\vec{\nabla} \psi-\frac{\vec{X}_{c, N_{f}}}{Z_{N_{f}}}\right)
\end{aligned}
$$

The reciprocal relations associated with the vector processes in (100) are

$$
\begin{array}{ll}
\mathrm{L}_{q v}=\mathrm{L}_{v q}, & \mathrm{~L}_{q e}=\mathrm{L}_{e q}, \quad \mathrm{~L}_{v e}=\mathrm{L}_{e v}, \\
\mathrm{~L}_{q i}=\mathrm{L}_{i q}, & \mathrm{~L}_{v i}=\mathrm{L}_{i v}, \quad \mathrm{~L}_{e i}=\mathrm{L}_{i e}, \quad i=1, \ldots, N_{f}-1, \\
\mathrm{~L}_{i j}=\mathrm{L}_{j i}, \quad i, j=1, \ldots, N_{f}-1, \quad i \neq j .
\end{array}
$$

The phenomenological equations and associated reciprocal relations for the chemical reactions are given by (72)-(74). With (102), the phenomenological equations for the vector processes, (100), contain $\frac{1}{2}\left(N_{f}+\right.$ $2)\left(N_{f}+3\right)$ independent phenomenological coefficients.

When the phenomenological equations are formed directly from the dissipation function given by (64), the following results are obtained for the vector fluxes:

$$
\begin{aligned}
& \vec{J}_{q}=-\mathrm{L}_{q q}^{\prime} \cdot \frac{\vec{\nabla} T}{T}+\mathrm{L}_{q v}^{\prime} \cdot \vec{X}_{p}+\sum_{i=1}^{N_{f}} \mathrm{~L}_{q i}^{\prime} \cdot \vec{X}_{c, i}-\mathrm{L}_{q e}^{\prime} \vec{\nabla} \psi, \\
& \vec{J}_{v}=-\mathrm{L}_{v q}^{\prime} \cdot \frac{\vec{\nabla} T}{T}+\mathrm{L}_{v v}^{\prime} \cdot \vec{X}_{p}+\sum_{i=1}^{N_{f}} \mathrm{~L}_{v i}^{\prime} \cdot \vec{X}_{c, i}-\mathrm{L}_{v e}^{\prime} \vec{\nabla} \psi,
\end{aligned}
$$




$$
\begin{aligned}
& \vec{J}_{i}^{\circ}=-\mathrm{L}_{i q}^{\prime} \cdot \frac{\vec{\nabla} T}{T}+\mathrm{L}_{i v}^{\prime} \cdot \vec{X}_{p}+\sum_{j=1}^{N_{f}} \mathrm{~L}_{i j}^{\prime} \cdot \vec{X}_{c, j}-\mathrm{L}_{i e}^{\prime} \vec{\nabla} \psi, \quad i=1, \ldots, N_{f} \\
& \vec{I}=-\mathrm{L}_{e q}^{\prime} \cdot \frac{\vec{\nabla} T}{T}+\mathrm{L}_{e v}^{\prime} \cdot \vec{X}_{p}+\sum_{i=1}^{N_{j}} \mathrm{~L}_{e i}^{\prime} \cdot \vec{X}_{c, i}-\mathrm{L}_{e e}^{\prime} \cdot \vec{\nabla} \psi
\end{aligned}
$$

We note that in (103d) the term containing $L_{c e}^{\prime}$ is Ohm's law. As noted before, the diffusional fluxes, $\vec{J}_{i}, i=1, \ldots, N_{f}$, in (103c) and the electrical fiux, $\vec{I}$, in (103d) are linearly dependent. Term by term comparison of the phenomenological equations given by (100) and (101) with those given by (103) provides the following relations between their coefficients:

$$
\begin{aligned}
& L_{q q}^{\prime}=L_{q q}, \quad L_{q v}^{\prime}=L_{q v}, \quad L_{v q}^{\prime}=L_{v q}, \quad L_{v v}^{\prime}=L_{v v}, \quad L_{e q}^{\prime}=L_{e e}, \\
& L_{q e}^{\prime}=L_{q e}, \quad L_{e q}^{\prime}=L_{e q}, \quad L_{v e}^{\prime}=L_{v e}, \quad L_{e v}^{\prime}=L_{e v}, \quad L_{e q}^{\prime} \\
& L_{q i}^{\prime}=L_{q i}, \quad L_{i q}^{\prime}=L_{i q}, \quad L_{v i}^{\prime}=L_{v i}, \quad L_{i v}^{\prime}=L_{i v}, \quad i=1, \ldots, N_{f}-1, \\
& L_{e i}^{\prime}=L_{e i}, \quad L_{i e}^{\prime}=L_{i e}, \quad i=1, \ldots, N_{f}-1, \\
& L_{i j}^{\prime}=L_{i j}, \quad i, j=1, \ldots, N_{f}-1, \quad i \neq j,
\end{aligned}
$$

and

$$
\begin{aligned}
& \mathrm{L}_{g N_{l}}^{\prime}=-\sum_{i=1}^{N_{f}-1} \frac{Z_{i}}{Z_{N_{l}}} \mathrm{~L}_{q i}+\frac{\mathrm{L}_{q \theta}}{Z_{N_{f}}}, \quad \mathrm{~L}_{N_{f}}^{\prime}=-\sum_{i=1}^{N_{f}-1} \frac{Z_{i}}{Z_{N_{j}}} \mathrm{~L}_{i q}+\frac{\mathrm{L}_{\ell q}}{Z_{N_{j}}} \\
& L_{v N_{f}}^{\prime}=-\sum_{i=1}^{N_{f}-1} \frac{Z_{i}}{Z_{N_{f}}} L_{v i}+\frac{L_{v e}}{Z_{N_{f}}}, \quad L_{N_{f} v}^{\prime}=-\sum_{i=1}^{N_{f}-1} \frac{Z_{i}}{Z_{N_{f}}} L_{i v}+\frac{L_{v v}}{Z_{N_{f}}}, \\
& \mathrm{~L}_{e N_{f}}^{\prime}=-\sum_{i=1}^{N_{f}-1} \frac{Z_{i}}{Z_{N_{f}}} \mathrm{~L}_{e i}+\frac{\mathrm{L}_{e e}}{Z_{N_{f}}}, \quad \mathrm{~L}_{N_{f e}}^{\prime}=-\sum_{i=1}^{N_{f}-1} \frac{Z_{i}}{Z_{N_{f}}} \mathrm{~L}_{i e}+\frac{\mathrm{L}_{e e}}{Z_{N_{f}}}, \\
& L_{i N_{j}}^{\prime}=-\sum_{j=1}^{N_{j}-1} \frac{Z_{i}}{Z_{N_{f}}} L_{i j}+\frac{L_{i e}}{Z_{N_{f}}}, \quad i=1, \ldots, N_{f}-1 \text {, } \\
& \mathrm{L}_{N_{f} i}^{\prime}=-\sum_{j=1}^{N_{j}-1} \frac{Z_{i}}{Z_{N_{j}}} \mathrm{~L}_{j i}+\frac{\mathrm{L}_{e i}}{Z_{N_{f}}}, \quad i=1, \ldots, N_{f}-1 \text {, } \\
& \mathrm{L}_{N_{f} N_{j}}^{\prime}=\sum_{i=1}^{N_{f}-1} \sum_{j=1}^{N_{j}-1} \frac{Z_{i} Z_{j}}{Z_{N_{f}}^{2}} \mathrm{~L}_{j i}-\sum_{i=1}^{N_{f}-1} \frac{Z_{i}}{Z_{N_{f}}^{2}}\left(\mathrm{~L}_{e i}+\mathrm{L}_{i e}\right)+\frac{\mathrm{L}_{e e}}{Z_{N_{f}}^{2}}
\end{aligned}
$$

Then, if the reciprocal relations in (102) are valid, (104) and (105) show that the reciprocal relations are valid also for the matrix of phenomenological coefficients, [ $\left.L^{\prime}\right]$, appearing in (103). As before, it is seen that, if (104) is used in (105), then the number of independent phenomenological coefficients in (103) is the same as in $(100)$, namely $\frac{1}{2}\left(N_{f}+2\right)\left(N_{f}+3\right)$.

If $(103 d)$ is solved for $\vec{\nabla} \psi$, the result is

$$
\vec{\nabla} \psi=-\left(\mathrm{L}_{e e}^{\prime}\right)^{-1} \cdot \mathrm{L}_{e q}^{\prime} \cdot \frac{\vec{\nabla} T}{T}+\left(\mathrm{L}_{e e}^{\prime}\right)^{-1} \cdot \mathrm{L}_{e v}^{\prime} \cdot \vec{X}_{p}+\left(\mathrm{L}_{e e}^{\prime}\right)^{-1} \cdot \sum_{i=1}^{N_{f}} \mathrm{~L}_{e i}^{\prime} \cdot \vec{X}_{c, i}-\left(\mathrm{L}_{e e}^{\prime}\right)^{-1} \cdot \vec{I}
$$

On the right-hand side of (106), the third term is called the diffusion potential, the first and third terms collectively are called the thermal diffusion potential, and the second term is called the streaming potential (DE Groot and MazUR, Chap. 13, §9; Chap. 15, §7). 


\section{CONCLUDING REMARKS}

The fundamental postulates of TTIP provide the basis for quantitative description of both direct and coupled irreversible processes. A considerable body of evidence supports the validity of the postulates in applications to transport processes. However, extension of the methods of TTIP to previously unstudied systems should be supported by experimental confirmation of the linearity of the phenomenological equations and validation of the appropriate Onsager reciprocal relations.

The phenomenological equations provide the necessary link between the fluxes appearing in the governing equations and the gradients of the thermodynamic variables, temperature, pressure, composition, gravitational potential, and electrical potential, that are the driving forces for the fluxes. Substitution of the phenomenological equations into the governing equations results in a system of nonlinear, mathematically coupled, partial differential equations for the thermodynamic variables.

A given system will be characterized by a fixed number of independent phenomenological coefficients, even though the phenomenological equations can be written in alternative forms, some involving dependencies among the fluxes. Identification of dependencies is very important in determining the minimum set of phenomenological coefficients (and accompanying phenomena) that will need to be measured in a laboratory experiment or specified in a numerical simulation. Careful formulation of the dissipation function for the system, as well as any side conditions (e.g., zero ionic current), will usually ensure that a correct set of phenomenological equations will be derived. The reciprocal relations provide an important constraint on the number of independent phenomenological coefficients needed to completely describe the system. In anisotropic systems, the phenomenological coefficients for transport processes will be second-rank tensors consisting of independent elements subject to experimental determination.

The governing equations and phenomenological equations derived here are intended to be general. Application to specific systems can result in considerable simplification. However, the nonlinear character of the governing equations persists in even the simplest systems, and solution of the equations requires numerical techniques. Part II of this set of papers (JACOBSEN AND Carnahan, 1990) demonstrates a method of solution for a particular application involving simultaneous variation of temperature, pressure, and composition.

Acknowledgemento-We thank Karsten Pruess for reviewing this paper and providing valuable comments and suggestions. This work was supported by the Director of Energy Research, Office of Basic Energy Sciences, Engineering and Geosciences Division, of the U. S. Department of Energy under Contract No. DE-AC03-76SF00098.

\section{REFERENCES}

Anderson D. E. and Graf D. L. (1976) Multicomponent electrolyte diffusion. Ann. Rev. Earth Plan. Sci. 4, 95-121.

BERRY F. A. F. (1967) Role of membrane filtration on origin of thermal brines, Imperial Valley, California. Am. Assoc. Pet. Geol. Bull. 51, 454-455.

Bredehoeft J. D., Blyth C. R., White W. A. and Maxey G. B. (1963) Possible mechanism for concentration of brines in subsurface formations. Am. Assoc. Pet. Geol. Bull. 47, 257-269.

Carnahan C. L. (1976) Non-equilibrium thermodynamics of groundwater flow systems: symmetry properties of phenomenological coefficients and considerations of hydrodynamic dispersion. J. Hydrology 31, 125-150.

Carnahan C. L. (1984) Thermodynamic coupling of heat and matter flows in near-field regions of nuclear waste repositories. In Scientific Basis for Nuclear Waste Management VII (ed. G. L. McVay), Materials Research Society Symposia Proceedings vol. 26, pp. 1023-1030. Elsevier Science Publishing. 
Carnahan C. L. (1985) Thermodynamically coupled mass transport processes in a saturated clay. In Scientific Basis for Nuclear Waste Management VIII (ed. C. M. JANTzen, J. A. Stone and R. C. EwING), Materials Research Society Symposia Proceedings Vol. 44, pp. 491-498. Materials Research Society.

Carnahan C. L. (1986) Thermal osmosis near a buried heat source. Int. Comm. Heat Mass Transfer 13, 659-664.

Coleman B. D. and Truesdell C. (1960) On the reciprocal relations of Onsager. J. Chem. Phys. 33, 28-31.

DE Groot S. R. and MAZUR P. (1962) Non-Equilibrium Thermodynamics. North-Holland. 510p.

DEMIR I. (1988) Studies of smectite membrane behavior: Electrokinetic, osmotic, and isotopic fractionation processes at elevated pressures. Geochim. Cosmochim. Acta 52, 727-737.

Denbiah K. (1971) The Principles of Chemical Equilibrium. Cambridge University Press. 494p.

Elrick D. E., Smiles D. E., Baumgartner M, and Groenevelt, P. H. (1976) Coupling phenomena in saturated homo-ionic montmorillonite: I. Experimental. Soil Sci. Soc. Am. J. 40, 490-491.

FrtTs D. D. (1962) Nonequilibrium Thermodynamics. McGraw-Hill. 173p.

Freeze R. A. and Cherry J. A. (1979) Groundwater. Prentice-Hall. 604p.

Fritz S. J., Hinz D. W. and Grossman E. L. (1987) Hyperfiltration-induced fractionation of carbon isotopes. Geochim. Cosmochim. Acte 51, 1121-1134.

Graf D. L. (1982) Chemical osmosis, reverse chemical osmosis, and the origin of subsurface brines. Geochim. Cosmochim. Acta 46, 1431-1448.

Greenberg J. A., Mitchell J. K. and Witherspoon P. A. (1973) Coupled salt and water flows in a groundwater basin. J. Geophys. Res. 78, 6341-6353.

Groenevelt P. H. and Bolt G. H. (1969) Non-equilibrium thermodynamics of the soil-water system. J. Hydrology 7, 358-388.

Groenevelt P. H. and ElRick D. E. (1976) Coupling phenomena in saturated homo-ionic montmorillonite: II. Theoretical. Soil Sci. Soc. Am. J. 40, 820-823.

Groenevelt P. H., Elrick D. E. and Blom T. J. M. (1976) Coupling phenomena in saturated homo-ionic montmorillonite: III. Analysis. Soil Sci. Soc. Am. J. 42, 671-674.

Groenevelt P. H., Elrick D. E. and LarYea K. B. (1976) Coupling phenomena in saturated homoionic montmorillonite: IV. The dispersion coefficient. Soil Sci. Soc. Am.'J. 44, 1168-1173.

Hafemann D. R. (1965) Charge separation in liquid junctions. J. Phys. Chem. 69, 4226-4231.

HASSANizadeh S. M. (1986a) Derivation of basic equations of mass transport in porous media, Part 1. Macroscopic balance laws. Adv. Water Resour. 9, 196-206.

HAssanizadeH S. M. (1986b) Derivation of basic equations of mass transport in porous media, Part 2. Generalized Darcy's and Fick's laws. Adv. Water Resour. 9, 207-222.

HASSANizAdeh S. M. and Leijnse T. (1988) On the modeling of brine transport in porous media. Water Resour. Res. 24, 321-330.

HAYDoN P. R. and GraF D. L. (1986) Studies of smectite membrane behavior: Temperature dependence, $20-180^{\circ} \mathrm{C}$. Geochim. Cosmochim. Acta 50, 115-121.

Jacobsen J. S. and Carnahan C. L. (1986) Chemical osmosis and thermal osmosis near a heat source buried in a saturated, semi-permeable medium (abstr.). Eos 67, 965.

Jacobsen J. S. and Carnahan C. L. (1990) Coupled transport processes in semipermeable media. Part II: Numerical methods and results. Lawrence Berkeley Laboratory Report $L B L-25619$. 
Katchalsky A. and CURRan P. F. (1967) Nonequilibrium Thermodynamics in Biophysics. Harvard University Press. 248p.

KHARAKA Y. K. and BerRY F. A. F. (1973) Simultaneous flow of water and solutes through geological membranes - I. Experimental investigation. Geochim. Cosmochim. Acta 37, 2577-2603.

LASAGA A. C. (1979) The treatment of multi-component diffusion and ion pairs in diagenetic fluxes. Am. J. Sci. 279, 324-346.

LETEY J. and KEMPER W. D. (1969) Movement of water and salt through a clay-water system: experimental verification of Onsager reciprocal relation. Soil Sci. Soc. Am. Proc. 33, 25-29.

Lewis G. N. and RANDall M. (1961) Thermodynamics, Second Edition (revised by K. S. PITzer and L. BREWER). McGraw-Hill. 723 p.

MARINE I. W. and FrITZ S. J. (1981) Osmotic model to explain anomalous hydraulic heads. Water Resour. Res. 17, 73-82.

MASoN E. A. (1974) The Onsager reciprocal relations - experimental evidence; discussion paper. In Foundations of Continu um Mechanics (eds. J. J. Delgado Domingos, M. N. R. NiNA and J. H. WhitelaW), pp. 215-227. MacMillan Press.

MiLleR D. G. (1956) Thermodynamic theory of irreversible processes I. The basic macroscopic concepts. Am. J. Phys. 24, 433-444.

MiLLER D. G. (1959) Ternary isothermal diffusion and the validity of the Onsager reciprocity relations. J. Phys. Chem. 63, 570-578.

MrLler D. G. (1960) Thermodynamics of irreversible processes. The experimental verification of the Onsager reciprocal relations. Chem. Rev. 60, 15-37.

MILLER D. G. (1965) Definitive test of the Onsager reciprocal relations in isothermal ternary diffusion of water-sodium chloride-potassium chloride. J. Phys. Chem. 69, 3374-3376.

MILLER D. G. (1966) Application of irreversible thermodynamics to electrolyte solutions. I. Determination of ionic transport coefficients $l_{i j}$ for isothermal vector transport processes in binary electrolyte systems. J. Phys. Chem. 70, 2639-2659.

MrLler D. G. (1974) The Onsager relations; experimental evidence. In Foundations of Continuum Mechanics (eds. J. J. Delgado Domingos, M. N. R. Nina and J. H. Whitelaw), pp. 185-214. MacMillan Press.

Miller D. G., RARD J. A., Eppstein L. B. and J. G. Albright (1984) Mutual diffusion coefficients and ionic transport coefficients $l_{i j}$ of $\mathrm{MgCl}_{2}-\mathrm{H}_{2} \mathrm{O}$ at $25^{\circ} \mathrm{C}$. J. Phys. Chem. 88, 5739-5748.

Milne I. H., McKelvey J. G. and Trump R. P. (1965) Semi-permeability of bentonite membranes to brines. Am. Assoc. Pet. Geol. Bull. 49, 103-105.

NeuziL C. E. (1986) Groundwater flow in low-permeability environments. Water Resour. Res. 22, 1163-1195.

OLSEN H. W. (1970) Simultaneous fluxes of liquid and charge in saturated kaolinite. Soil Sci. Soc. Am. Proc. 33, 338-344.

Onsager L. (1931) Reciprocal relations in irreversible processes. I. Phys. Rev. 37, 405-426; II. Phys. Rev. 38, 2265-2279.

Phillips F. M. and Bentley H. W. (1987) Isotopic fractionation during ion filtration: I. Theory. Geochim. Cosmochim. Acta 51, 683-695.

REED W. E. (1970) Transport of water away from a buried heat source with special reference to hydrologic phenomena observed at Aardvark nuclear detonation. J. Geophys. Res. 75, 415-429. 
Sharland S. M., Tasker P. W. and Tweed C. J. (1987) The coupling of chemical and transport processes in near-field modeling. In Scientific Basis for Nuclear Waste Management $X$ (eds. J. K. Bates and W. B. SeefeldT), Materials Research Society Symposia Proceedings, Vol. 84, pp. 683-694. Materials Research Society.

SRivastava R. C. and ABraham M. G. (1979) Electro-osmotic permeability of composite clay membranes. J. Non-Equilib. Thermodyn. 4, 107-118.

SRIVastava R. C. and AVASTH P. K. (1975) Non-equilibrium thermodynamics of thermo-osmosis of water through kaolinite. J. Hydrology 24, 111-120.

Young A. and Low P. F. (1965) Osmosis in argillaceous rocks. Am. Assoc. Pet. Geol. Bull. 49, 1004-1008.

\section{APPENDIX A: DERIVATION OF EQUATIONS (11) AND (12)}

The total differentials of the entropies of each phase are, using temperature, volume, and composition as independent variables,

$$
\begin{aligned}
& d S_{f}=\left(\frac{\partial S_{f}}{\partial T}\right)_{V, m} d T+\left(\frac{\partial S_{f}}{\partial V}\right)_{T, m} d V_{f}+\sum_{i=0}^{N_{f}}\left(\frac{\partial S_{f}}{\partial m_{i}}\right)_{T, V_{, m_{k}}} d m_{i}, \\
& d S_{j}=\left(\frac{\partial S_{j}}{\partial T}\right)_{V, m} d T+\left(\frac{\partial S_{j}}{\partial V}\right)_{T, m} d V_{j}+\left(\frac{\partial S_{j}}{\partial m_{j}}\right)_{T, V} d m_{j}, j=1, \ldots, N_{r}, \\
& d S_{n}=\left(\frac{\partial S_{n}}{\partial T}\right)_{V, m} d T+\left(\frac{\partial S_{n}}{\partial V}\right)_{T, m} d V_{n},
\end{aligned}
$$

and, using pressure, volume, and composition as independent variables,

$$
\begin{aligned}
d S_{f} & =\left(\frac{\partial S_{f}}{\partial P}\right)_{V, m} d P+\left(\frac{\partial S_{f}}{\partial V}\right)_{P, m} d V_{f}+\sum_{i=0}^{N_{f}}\left(\frac{\partial S_{f}}{\partial m_{i}}\right)_{P, V_{,} m_{k}} d m_{i}, \\
d S_{j} & =\left(\frac{\partial S_{j}}{\partial P}\right)_{V, m} d P+\left(\frac{\partial S_{j}}{\partial V}\right)_{P, m} d V_{j}+\left(\frac{\partial S_{j}}{\partial m_{j}}\right)_{P, V} d m_{j}, j=1, \ldots, N_{r}, \\
d S_{n} & =\left(\frac{\partial S_{n}}{\partial P}\right)_{V, m} d P+\left(\frac{\partial S_{n}}{\partial V}\right)_{P, m} d V_{n},
\end{aligned}
$$

where the subscripts on the partial derivatives indicate which variables are held constant during differentiation. In (A-1C) and (A-2c) the mass of the nonreactive solid phase was assumed constant.

Under the postulate of local equilibrium, if $\mathcal{Q}$ represents any extensive property such as entropy, $S$, or volume, $V$, the partial specific value of the property, $\overline{\mathcal{Q}}_{i}$, for the $i$-th substance is intensive and is defined by

$$
\overline{\boldsymbol{Q}}_{i}=\left(\frac{\partial \mathcal{Q}}{\partial m_{i}}\right)_{T, P, m_{k}}
$$

where $m_{i}$ is the mass of substance $i$ and the differentiation is done while keeping masses $m_{k}$ of all other substances constant; for a pure substance, the partial specific value is identical to the specific value (DENBIGH, 1971, p. 101, 103). Then, differentiating (A-1a) and (A-2a) with respect to $m_{i}$, keeping $T$, $P$, and $m_{k}$ for $i \neq k$ constant, yields (DENBIGH, 1971, p. 92)

$$
\bar{S}_{i}=\left(\frac{\partial S_{f}}{\partial V}\right)_{T, m} \bar{V}_{i}+\left(\frac{\partial S_{f}}{\partial m_{i}}\right)_{T, V, m_{k}}, i=0, \ldots, N_{f}
$$

and 


$$
\bar{S}_{i}=\left(\frac{\partial S_{f}}{\partial V}\right)_{P, m} \nabla_{i}+\left(\frac{\partial S_{f}}{\partial m_{i}}\right)_{P, V, m_{h}}, i=0, \ldots, N_{f}
$$

Similar expressions may be written for the result of differentiating (A-1b) and (A-2b) with respect to $m_{j}$ :

$$
s_{j}=\left(\frac{\partial S_{j}}{\partial V}\right)_{T, m} v_{j}+\left(\frac{\partial S_{j}}{\partial m_{j}}\right)_{T, V}, j=1, \ldots, N_{r}
$$

and

$$
s_{j}=\left(\frac{\partial S_{j}}{\partial V}\right)_{P, m} v_{j}+\left(\frac{\partial S_{j}}{\partial m_{j}}\right)_{P, V} \cdot j=1, \ldots, N_{r}
$$

where $s_{j}$ is the specific entropy and $v_{j}$ the specific volume of solid $j$.

The following thermodynamic identities may be used to eliminate the partial derivatives from (A-1), (A-2), (A-4) and (A-5) (DenBigh, 1971, pp. 91, 96-97):

$$
\begin{aligned}
& \left(\frac{\partial S_{k}}{\partial T}\right)_{V, m}=\frac{\rho_{k} c_{v, k}}{T} \epsilon_{k} V, \\
& \left(\frac{\partial S_{k}}{\partial V}\right)_{T, m}=\left(\frac{\partial P}{\partial T}\right)_{V, m}=-\left(\frac{\partial P}{\partial V}\right)_{T, m}\left(\frac{\partial V}{\partial T}\right)_{P, m}=\frac{\beta_{k}}{\kappa_{k}}, \\
& \left(\frac{\partial S_{k}}{\partial P}\right)_{V, m}=\left(\frac{\partial S_{k}}{\partial T}\right)_{V, m}\left(\frac{\partial T}{\partial P}\right)_{V, m}=\frac{\rho_{k} c_{v, k}}{T} \frac{\kappa_{k}}{\beta_{k}} \epsilon_{k} V, \\
& \left(\frac{\partial S_{k}}{\partial V}\right)_{P, m}=\left(\frac{\partial S_{k}}{\partial T}\right)_{P, m}\left(\frac{\partial T}{\partial V}\right)_{P, m}=\frac{\rho_{k} c_{p, k}}{\beta_{k} T}
\end{aligned}
$$

Substituting (A-7) into (A-4a) and (A-5a) and substituting (A-9) into (A-4b) and (A-5b) yield

$$
\begin{aligned}
& \left(\frac{\partial S_{f}}{\partial m_{i}}\right)_{T, V, m_{k}}=\bar{S}_{i}-\frac{\beta_{f}}{\kappa_{f}} \bar{V}_{i}, \quad i=0, \ldots, N_{f}, \\
& \left(\frac{\partial S_{f}}{\partial m_{i}}\right)_{P, V, m_{k}}=\bar{S}_{i}-\frac{\rho_{f} c_{p, f}}{\beta_{f} T} \bar{V}_{i}, \quad i=0, \ldots, N_{f}, \\
& \left(\frac{\partial S_{j}}{\partial m_{j}}\right)_{T, V}=s_{j}-\frac{\beta_{j}}{\kappa_{j}} v_{j}, \quad j=1, \ldots, N_{r}, \\
& \left(\frac{\partial S_{j}}{\partial m_{j}}\right)_{P, V}=s_{j}-\frac{c_{p, j}}{\beta_{j} T}, \quad j=1, \ldots, N_{r},
\end{aligned}
$$

where subscripts $f$ and $j$ refer to the fluid and the $j$-th solid phase, respectively, and the relation, $\rho_{j} v_{j}=1, j=1, \ldots, N_{r}$, was used in (A-11b). Finally, substituting (A-1) into (10) and then (A-2) into (10), using (A-6)-(A-11), and noting that $d V_{k}=\epsilon_{k} d V$ for phase $k$ give (11) and (12).

\section{APPENDIX B: DERIVATION OF EQUATIONS (15) AND (16)}

Substituting (14) into (11) and (12) give

$$
d\left(V C_{s}\right)=\Gamma_{1} \frac{V}{T} d T+\Gamma_{2} d V+\sum_{i=0}^{N_{j}}\left(\bar{S}_{i}-\frac{\beta_{f}}{\kappa_{f}} \bar{V}_{i}\right) d\left(\epsilon_{f} V C_{i}\right)+\sum_{j=1}^{N_{r}}\left(s_{j}-\frac{\beta_{j}}{\kappa_{j}} v_{j}\right) d\left(V C_{j}\right)
$$




$$
d\left(V C_{\imath}\right)=\Lambda_{1} \frac{V}{T} d P+\frac{\Lambda_{2}}{T} d V+\sum_{i=0}^{N_{f}}\left(\bar{S}_{i}-\frac{\rho_{f} c_{p, f}}{\beta_{f} T} \bar{V}_{i}\right) d\left(\epsilon_{j} V C_{i}\right)+\sum_{j=1}^{N_{r}}\left(s_{j}-\frac{c_{p, j}}{\beta_{j} T}\right) d\left(V C_{j}\right)
$$

Integral forms of the entropy equations may be obtained using Euler's theorem on homogeneous functions (Katchalsky and Curran, 1967, pp. 22-23; Denbigh, 1971, pp. 92-93). Thus, we have at constant temperature,

$$
C_{\imath}=\Gamma_{2}+\sum_{i=0}^{N_{f}}\left(\bar{S}_{i}-\frac{\beta_{f}}{\kappa_{f}} \bar{V}_{i}\right) \epsilon_{f} C_{i}+\sum_{j=1}^{N_{r}}\left(\varepsilon_{j}-\frac{\beta_{j}}{\kappa_{j}} v_{j}\right) C_{j}
$$

and at constant pressure,

$$
C_{\imath}=\frac{\Lambda_{2}}{T}+\sum_{i=0}^{N_{f}}\left(\bar{S}_{i}-\frac{\rho_{f} c_{p, f}}{\beta_{f} T} \nabla_{i}\right) \epsilon_{f} C_{i}+\sum_{j=1}^{N_{r}}\left(s_{j}-\frac{c_{p, j}}{\beta_{j} T}\right) C_{j}
$$

Returning to (B-1) and.(B-2), expanding the differential terms and collecting factors of $d V$ and $V$ yield

$$
\begin{aligned}
& \left\{C_{s}-\Gamma_{2}-\sum_{i=0}^{N_{f}}\left(\bar{S}_{i}-\frac{\beta_{f}}{\kappa_{f}} \bar{V}_{i}\right) \epsilon_{f} C_{i}-\sum_{j=1}^{N_{F}}\left(s_{j}-\frac{\beta_{j}}{\kappa_{j}} v_{j}\right) C_{j}\right\} d V \\
& =\left\{-d C_{i}+\frac{\Gamma_{1}}{T} d T+\sum_{i=0}^{N_{f}}\left(\bar{S}_{i}-\frac{\beta_{f}}{\kappa_{f}} \nabla_{i}\right) d\left(\epsilon_{f} C_{i}\right)+\sum_{j=1}^{N_{r}}\left(s_{j}-\frac{\beta_{j}}{\kappa_{j}} v_{j}\right) d C_{j}\right\} V, \\
& \left\{C_{i}-\frac{\Lambda_{2}}{T}-\sum_{i=0}^{N_{f}}\left(\bar{S}_{i}-\frac{\rho_{f} c_{p, f}}{\beta_{f} T} \bar{V}_{i}\right) \epsilon_{f} C_{i}-\sum_{j=1}^{N_{r}}\left(s_{j}-\frac{c_{p, j}}{\beta_{j} T}\right) C_{j}\right\} d V \\
& =\left\{-d C_{i}+\frac{\Lambda_{1}}{T} d P+\sum_{i=0}^{N_{f}}\left(S_{i}-\frac{\rho_{\rho} c_{p, j}}{\beta_{f} T} \nabla_{i}\right) d\left(\epsilon_{f} C_{i}\right)+\sum_{j=1}^{N_{r}}\left(s_{j}-\frac{c_{p, j}}{\beta_{j} T}\right) d C_{j}\right\} V .
\end{aligned}
$$

By (B-3) and (B-4), the left-hand sides of (B-5) and (B-6) are zero. Using these results, forming the time derivatives of the right-hand sides of (B-5) and (B-6), allowed by the postulate of local equilibrium, and multiplying both sides of each equation by $T$ give the results (15) and (16).

\section{APPENDIX C: DERIVATION OF EQUATIONS (17), (20), AND (21)} 1969):

The Gibbs equation for a volume $V$ of the saturated porous matrix is (Groenevelt and BolT,

$$
d E=T d S-P d V+\sum_{i=0}^{N_{j}}\left(\mu_{i}+\varphi_{i}\right) d m_{i}+\sum_{j=1}^{N_{r}}\left(\mu_{j}+\varphi_{j}\right) d m_{j}
$$

where $E$ is the total static energy (internal energy plus potential energy) and $S$ is the entropy of the saturated porous matrix. Using Euler's theorem on homogeneous functions (DENBIGH, 1971, p. 92), the 
integrated form of (C-1) is

$$
E=T S-P V+\sum_{i=0}^{N_{f}}\left(\mu_{i}+\varphi_{i}\right) m_{i}+\sum_{j=1}^{N_{r}}\left(\mu_{j}+\varphi_{j}\right) m_{j}
$$

Replacing the extensive quantities in (C-1) and (C-2) by their intensive counterparts results in the relations,

$$
d\left(V C_{e}\right)=T d\left(V C_{\imath}\right)-P d V+\sum_{i=0}^{N_{j}}\left(\mu_{i}+\varphi_{i}\right) d\left(\epsilon_{j} V C_{i}\right)+\sum_{j=1}^{N_{r}}\left(\mu_{j}+\varphi_{j}\right) d\left(V C_{j}\right)
$$

and

$$
C_{e}=T C_{,}-P+\sum_{i=0}^{N_{j}}\left(\mu_{i}+\varphi_{i}\right) \epsilon_{j} C_{i}+\sum_{j=1}^{N_{\tau}}\left(\mu_{j}+\varphi_{j}\right) C_{j}
$$

Expanding the differentials in (C-3), using (C-4), forming time derivatives, and rearranging terms yield (17).

Using (19), (18) is substituted into (17). The result is substituted into (15) and (16), and the time derivatives of the mass concentrations are replaced by (4), using (5). This yields

$$
\begin{aligned}
\Gamma_{1} \frac{\partial T}{\partial t}= & -\vec{\nabla} \cdot\left[\vec{J}_{q}+\sum_{i=0}^{N_{f}} \vec{J}_{i}\left(\bar{H}_{i}+\varphi_{i}\right)\right]-\sum_{i=0}^{N_{f}}\left(\mu_{i}+\varphi_{i}+T \vec{S}_{i}-\frac{\beta_{f} T}{\kappa_{f}} \nabla_{i}\right)\left(-\vec{\nabla} \cdot \vec{J}_{i}+A_{i f}+A_{i r}\right) \\
& -\sum_{j=1}^{N_{r}}\left(\mu_{j}+\varphi_{j}+T s_{j}-\frac{\beta_{j} T}{\kappa_{j}} v_{j}\right) A_{j r} \\
\Lambda_{1} \frac{\partial P}{\partial t}= & -\vec{\nabla} \cdot\left[\vec{J}_{q}+\sum_{i=0}^{N_{f}} \vec{J}_{i}\left(\bar{H}_{i}+\varphi_{i}\right)\right]-\sum_{i=0}^{N_{f}}\left(\mu_{i}+\varphi_{i}+T \vec{S}_{i}-\frac{\rho_{f} c_{p, f}}{\beta_{f}} \bar{V}_{i}\right)\left(-\vec{\nabla} \cdot \vec{J}_{i}+A_{i f}+A_{i r}\right) \\
& -\sum_{j=1}^{N_{r}}\left(\mu_{j}+\varphi_{j}+T s_{j}-\frac{c_{p, j}}{\beta_{j}}\right) A_{j r}
\end{aligned}
$$

Using the thermodynamic relations,

$$
\begin{gathered}
\mu_{i}=\bar{H}_{i}-T \bar{S}_{i}, \quad i=0, \ldots, N_{f}, \\
\mu_{j}=h_{j}-T s_{j}, \quad j=1, \ldots, N_{r},
\end{gathered}
$$

where $h_{j}$ is the specific enthalpy of solid $j$, and rearranging terms give (20) and (21). 